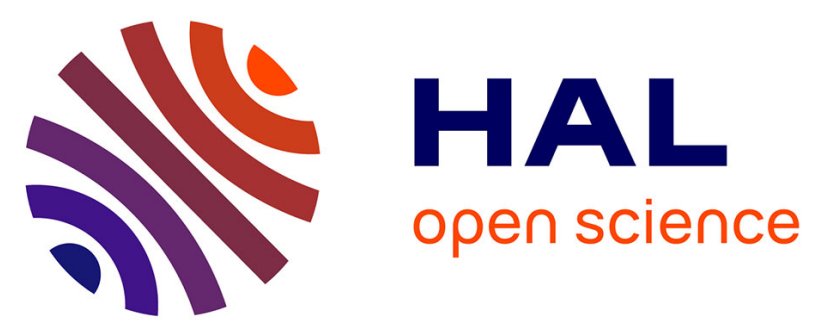

\title{
Whistler envelope solitons. II. Interaction with non-relativistic electron beams in plasmas with density inhomogeneities
}

\author{
C. Krafft, A. S. Volokitin
}

\section{- To cite this version:}

C. Krafft, A. S. Volokitin. Whistler envelope solitons. II. Interaction with non-relativistic electron beams in plasmas with density inhomogeneities. Physics of Plasmas, 2018, 25 (10), pp.102302. 10.1063/1.5041075 . hal-01992855

\section{HAL Id: hal-01992855 \\ https://hal.sorbonne-universite.fr/hal-01992855}

Submitted on 24 Jan 2019

HAL is a multi-disciplinary open access archive for the deposit and dissemination of scientific research documents, whether they are published or not. The documents may come from teaching and research institutions in France or abroad, or from public or private research centers.
L'archive ouverte pluridisciplinaire HAL, est destinée au dépôt et à la diffusion de documents scientifiques de niveau recherche, publiés ou non, émanant des établissements d'enseignement et de recherche français ou étrangers, des laboratoires publics ou privés. 
Whistler envelope solitons. II. Interaction with non relativistic electron beams in plasmas with density inhomogeneities

C. $\mathrm{Krafft}^{1}$ and A. Volokitin ${ }^{2,3}$

1) Laboratoire de Physique des Plasmas, CNRS, Ecole polytechnique, UPMC Univ. Paris 06, Univ. Paris-Sud, Observatoire de Paris, Université Paris-Saclay, Sorbonne Universités, PSL Research University, 91128 Palaiseau, France

2)IZMIRAN, Troitsk, 142190, Moscow, Russia

3) Space Research Institute (IKI) 117997, 84/32 Profsoyuznaya Str, Moscow, Russia

The paper studies the self-consistent interactions between whistler envelope solitons and electron beams in inhomogeneous plasmas, using a Hamiltonian model of waveparticle interaction where nonlinear equations describing the dynamics of whistler and ion acoustic waves and including a beam current term are coupled with Newton equations. It allows to describe the parallel propagation of narrowband whistlers interacting with arbitrary particle distributions in irregular plasmas. It is shown that the whistler envelope soliton does not exchange energy with all the resonant electrons as in the case of whistler turbulence but mostly with those moving in its close vicinity (locality condition), even if the downstream particle distribution is perturbed. During these interactions the soliton can either damp and accelerate particles, either absorb beam energy and cause electron deceleration. If the energy exchanges are significative, the envelope is deformed; its upstream front can steepen whereas oscillations can appear on its downstream side. Weak density inhomogeneities as the random fluctuations of the solar wind plasma have no strong impact on the interactions of the whistler soliton with the resonant particles. 


\section{INTRODUCTION}

Interactions between whistler waves and electron fluxes have been extensively studied owing to space measurements ${ }^{2-7}$ and laboratory experiments ${ }^{8-12}$, as well as in analytical

works and numerical simulations ${ }^{13-23}$. Moreover, the interactions of whistlers with particles in inhomogeneous plasmas have been investigated theoretically and numerically. For example, such interactions were studied ${ }^{24}$ in a plasma with a non uniform magnetic field, considering parallel propagating coherent whistler waves interacting with energetic resonant electrons. A lot of studies were focused on the problem of whistler chorus emissions where the inhomogeneity of the ambient magnetic field plays an important role. However, the influence of other types of inhomogeneities, as random plasma density irregularities, on whistlers' interactions with electron fluxes were only rarely investigated, in particular during the occurrence of nonlinear effects; such plasma irregularities are present in the solar wind or in other space plasmas. They are particularly important as they can strongly reduce the efficiency of wave-particle interactions, as it was evidenced recently for the case of Langmuir turbulence in the solar wind ${ }^{25,26}$.

Measurements by the satellite Helios have shown that whistler turbulence with wave frequencies up to the electron gyrofrequency is present in the solar wind, together with the occurrence of ion acoustic-like type oscillations ${ }^{27}$. This observation was also confirmed by the spacecraft Ulysses $^{28}$ which detected an ubiquitous whistler wave background in the solar wind, that can be the source of many wave-particle phenomena. When the whistler waves' intensities become sufficiently large, nonlinear effects can occur, and in particular modulational instabilities which can give rise to soliton formation. Large amplitude whistlers have been measured by the Wind and Stereo satellites ${ }^{5}$ and a statistical study of such waves and their association with energetic electron distributions was reported ${ }^{7}$. Localized whistler wave packets were observed in the solar wind ${ }^{29}$ and the authors suggested they could be soliton structures. Such packets were also detected by the spacecraft Freja and Cluster in connection with density cavities, and were reported to be whistler envelope solitons ${ }^{30,31}$. At the same time, satellites detected different distributions of energetic particles in the plasma regions where such observations were performed. Consequently the questions arise under what conditions these whistler envelope solitons are able to interact with particles, whether or not they can exchange a noticeable amount of energy and momentum with them and in 
what extent they can loose their stability.

The interactions of Langmuir and ion acoustic solitons with electron fluxes have been studied theoretically and numerically in non magnetized plasmas by different authors ${ }^{32-37}$. They have shown that the solitons can damp and that, as a consequence, the kinetic energy of the resonant particles' population can increase. For electromagnetic waves in a magnetized plasma, the situation is obviously more complex and only very rare works have been undergone on this subject. To our knowledge, only in Ref. 37 the case of whistler solitons interacting with an electron beam is investigated; however the authors limited their study to the case of whistlers with frequencies very small compared to the electron gyrofrequency and a parallel beam velocity distribution modeled by a Dirac function. They only calculated the rate of energy loss of the solitons to the beam in conditions typical for the solar wind, using an approach involving a gas of solitons; they did not consider the possibility for the soliton to gain energy from the particles and did not perform numerical simulations to study the dynamics of the whistler envelope solitons interacting with the electrons.

In view of the above, we have built a Hamiltonian model describing the interaction of whistler waves with electron fluxes in a inhomogeneous magnetized plasma, in order to perform numerical simulations aimed to study various nonlinear phenomena. The model involves in particular a parabolic-type equation including self-consistently a beam current term, which is coupled to Newton equations describing the individual motion of the beam electrons as well as to lower frequency equations taking into account ponderomotive effects ${ }^{42}$. This paper is aimed to present this model in detail and to apply it to study the interactions of whistler envelope solitons with electron fluxes (in homogeneous or inhomogeneous plasmas), i.e. to understand the dynamics, the stability and the energy exchanges of the soliton during its interactions with the resonant particles. Such model can also be used to understand whistler chorus modulation by density variations. As a first approach, we consider hereafter coherent narrowband whistler waves propagating parallel to the ambient magnetic field and interacting with arbitrary electron distributions.

\section{LINEAR EXCITATION OF COHERENT WHISTLERS BY BEAMS}

Most of whistler waves' instabilities are driven by the free energy contained in anisotropic electron velocity distributions as loss cones, rings, horseshoes or beams, which is released via 
normal cyclotron resonant interactions, as well as in distributions presenting perpendicular temperatures larger than parallel ones. For example, in the solar wind, the temperature anisotropy and the electron heat flux instabilities of whistlers can likely occur.

Our study concerns narrowband whistler waves excited by beams and propagating parallel to the ambient magnetic field. In this view, studying the wave emission of a hot anisotropic electron beam drifting along a constant magnetic field in a homogeneous cold plasma for a wide range of parameters, some authors ${ }^{16}$ calculated the variation of the maximum growth rates $\gamma_{\max }$ of all types of waves emitted as a function of their propagation angle $\theta$, showing that for the electromagnetic whistler mode, $\gamma_{\max }$ reaches its highest value at parallel propagation $\theta=0^{\circ}$ and remains almost unchanged when $\theta$ increases until $30^{\circ}$. However, the electrostatic mode excited (with phase velocity in the direction of the drifting electron beam) presents a larger growth rate for $\theta=0^{\circ}$, even if it decreases strongly with $\theta$ to reach, around $\theta \simeq 30^{\circ}$, roughly the same value as that of the electromagnetic mode. So, for quasi-parallel propagation $\left(\theta \lesssim 30^{\circ}\right)$, both the whistler and the electrostatic modes can be excited simultaneously. On the contrary, for strict parallel propagation, electrostatic waves grow more fast, so that the beam electrons are trapped and diffused, reducing by the way significantly the whistler growth rates. However, the whistler mode is excited via the normal cyclotron resonances and the electrostatic one by the Landau resonances; therefore, if the beam is not monoenergetic but warm, both modes, excited on different time scales, are also characterized by different ranges of wavenumbers and frequencies. Indeed, at parallel propagation, the electrostatic and electromagnetic modes are uncoupled as they act on different time scales, having different growth rates. But at oblique propagation their growth rates can become comparable and they can act simultaneously.

In Ref. ${ }^{39}$ it was shown that a cold beam should not be able to excite efficiently whistler waves, due to the fact that electrostatic waves grow more fast than whistlers. However, a sufficiently warm beam is able to radiate whistlers in spite of the diminution of their growth rates during the quasi-linear electrostatic diffusion process. Moreover they estimated that for a dense plasma the whistler instability can dominate if the beam pitch angle $\theta_{p}=$ $\tan ^{-1}\left(\left\langle v_{\perp}^{2}\right\rangle / v_{b}^{2}\right)^{1 / 2}$ is large enough, i.e. if the perpendicular thermal velocity is large enough $\left(v_{b}\right.$ is the beam parallel velocity and $\left\langle v_{\perp}^{2}\right\rangle$ is the square perpendicular velocity proportional to the perpendicular thermal energy). For warm beams, the wavenumber width $\Delta k$ wherein the whistler growth rates are positive can be rather narrow (i.e. $\Delta k / k \ll 1$ ), as shown below (see 
also Refs. $\left.{ }^{14-16}\right)$. In this case the growth of narrowband whistlers occurs for a limited range of frequencies and wavenumbers; note that, for such type of emissions, the ranges of $k$ where normal cyclotron and Landau resonant velocities are lying are narrow and, for adequate conditions, they can be well separated one from the other. This circumstance justifies the possibility to consider intense parallel propagating whistler emissions and assume hereafter that $\Delta k / k \lesssim 0.1$.

Approximate expressions for the linear growth rate of whistler waves excited in a cold plasma by a warm anisotropic non relativistic beam of velocity distribution function $F_{b}\left(v_{z}, v_{\perp}\right)$ were determined ${ }^{40}$, neglecting the parallel temperature effects compared to the perpendicular ones. The parallel propagating whistler dispersion relation can be written as follows, when the beam parallel thermal velocity $v_{T_{b z}}$ is very small compared to the resonance velocity $v_{R}=\left(\omega-\omega_{c}\right) / k$ (i.e. $\left.k v_{T_{b z}} /\left(\omega-\omega_{c}\right) \ll 1\right)$

$c^{2} k^{2}-\omega^{2}+\omega_{p}^{2} \frac{\omega}{\omega-\omega_{c}}+\frac{n_{b}}{n_{0}} \omega_{p}^{2}\left(\frac{\omega-k v_{b}}{\omega-k v_{b}-\omega_{c}}+\frac{k^{2}\left\langle v_{\perp}^{2}\right\rangle}{2\left(\omega-k v_{b}-\omega_{c}\right)^{2}}+\frac{k^{2}\left\langle v_{z}^{2}\right\rangle \omega_{c}}{2\left(\omega-k v_{b}-\omega_{c}\right)^{3}}\right) \simeq 0$,

where $n_{b}$ and $n_{0}$ are the beam and the plasma densities; $\omega$ and $k$ are the frequency and the wavenumber of the whistler; $\omega_{p}$ and $\omega_{c}$ are the electron plasma and cyclotron frequencies; $\left\langle v_{z}^{2}\right\rangle=\int_{-\infty}^{\infty} F_{z}\left(v_{z}\right) v_{z}^{2} d v_{z}$ and $\left\langle v_{\perp}^{2}\right\rangle=2 \pi \int_{0}^{\infty} F_{\perp}\left(v_{\perp}\right) v_{\perp}^{3} d v_{\perp}$ are the mean square velocities along and perpendicular to the magnetic field, respectively, where $F_{z}$ and $F_{\perp}$ are the parallel and the perpendicular beam velocity distributions. This equation has been solved numerically (see Fig. 1) when $F_{b}\left(v_{z}, v_{\perp}\right)$ is a DGH (Dory-Guest-Harris) function ${ }^{41}$ of index $\nu$

$$
F_{b}\left(v_{z}, v_{\perp}\right)=\frac{1}{\pi^{3 / 2} \Gamma(\nu / 2+1)} \frac{v_{\perp}^{\nu}}{v_{T_{b z}} v_{T_{b \perp}}^{\nu+2}} \exp \left(-\frac{v_{\perp}^{2}}{v_{T_{b \perp}}^{2}}\right) \exp \left(-\frac{\left(v_{z}-v_{b}\right)^{2}}{v_{T_{b z}}^{2}}\right)=F_{z}\left(v_{z}\right) F_{\perp}\left(v_{\perp}\right),
$$

where $v_{T_{b \perp}}$ is the perpendicular beam thermal velocity, with $2 \pi \int_{0}^{\infty} F_{\perp}\left(v_{\perp}\right) v_{\perp} d v_{\perp}=1$ and $\int_{-\infty}^{\infty} F_{z}\left(v_{z}\right) d v_{z}=1 ; \nu$ is an integer and $\Gamma$ is the Gamma function.

The beam drifts in the direction opposite to the ambient magnetic field $\mathbf{B}_{0}=B_{0} \mathbf{z}$. The interactions between the beam and the waves take place at normal cyclotron resonance conditions $k v_{z}=\omega-\omega_{c}$. Using (2) one can calculate that $\left\langle v_{\perp}^{2}\right\rangle=v_{T_{b \perp}}^{2}(\nu / 2+1)$. We define in this case the anisotropy factor as $A=(\nu+1) T_{b \perp} / T_{b z}$, which reduces to $A=T_{b \perp} / T_{b z}$ for a bi-Maxwellian, where $T_{b z}$ and $T_{b \perp}$ are the parallel and the perpendicular beam temperatures.

Figure 1 shows the variations of the growth rate $\gamma / \omega_{c}$ of whistler waves as a function of their normalized wavenumber $c k / \omega_{c}$, for three different anisotropy factors $A$ and typical 

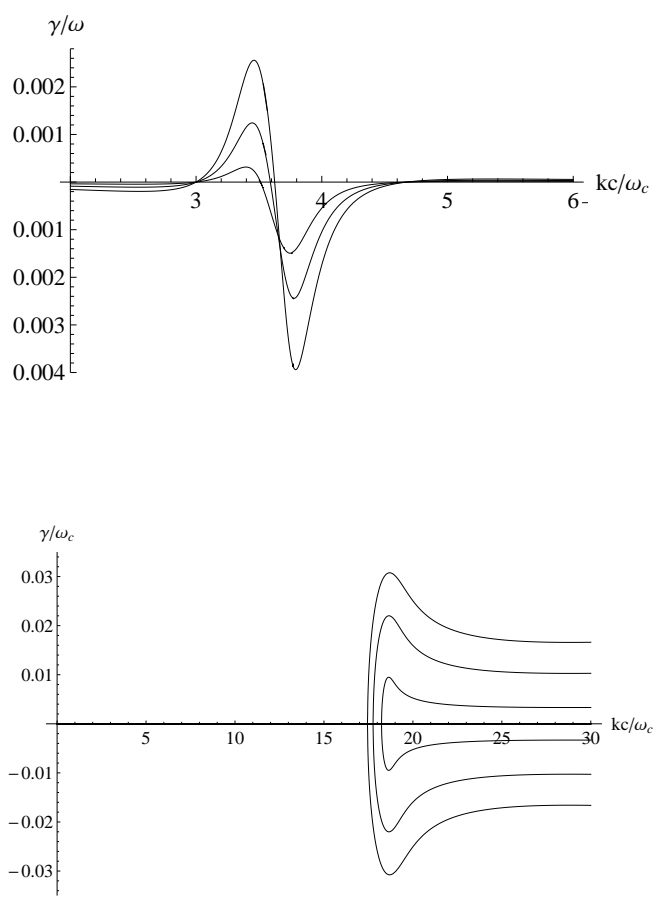

FIG. 1. Linear growth rates $\gamma / \omega_{c}$ of whistlers as a function of their wavenumbers $c k / \omega_{c}$, for different temperature anisotropy factors $A$, in the case of a DGH function with $\nu=1$. (Upper panel) Magnetospheric conditions with $\omega_{p} / \omega_{c}=5, n_{b} / n_{0}=0.0001, v_{b} / v_{T}=-18, \omega_{0} / \omega_{c} \simeq 0.32$, $T_{e}=50 \mathrm{eV}, A=50,100,200$. (Lower panel) Solar wind conditions with $\omega_{p} / \omega_{c}=100, n_{b} / n_{0}=$ $0.00005, v_{b} / v_{T}=-8, \omega_{0} / \omega_{c} \simeq 0.03, T_{e}=20 e V, A=3,30,80$. For both cases, the maximum growth rate $\gamma_{\max } / \omega_{c}$ increases with $A$. $T_{e}$ and $v_{T}$ are the electron plasma temperature and the corresponding thermal velocity, respectively.

conditions of the Earth magnetosphere (upper panel) and the solar wind (lower panel). The maximum growth rate increases with $A$. One can see in both cases that the wavenumber region $\Delta k$ where $\gamma / \omega_{c}$ is maximum is narrow, as $\Delta k / k \lesssim 0.1$. Therefore coherent narrowband whistlers propagating parallel to the ambient magnetic field can be efficiently excited by a warm anisotropic beam. Note that the maximum growth rates can reach values of the order of $\gamma / \omega_{c} \simeq 0.01$ even for weak beams $\left(n_{b} / n_{0} \ll 10^{-4}\right)$ and rather small anisotropies. Then it is of interest to develop a nonlinear model of coherent whistlers which takes into account the interactions of electromagnetic waves with particle fluxes together with different nonlinear effects : waves' interactions with plasma inhomogeneities, soliton propagation, wave decay, etc. Such model is developed in the next Section. 


\section{NONLINEAR HAMILTONIAN MODEL}

Let us build a model describing the self-consistent interactions of coherent electromagnetic whistlers with particles in a plasma presenting inhomogeneities, by following the approach developed in our companion paper ${ }^{42}$. The electric field of the whistler wave is given by

$$
\mathbf{E}=\operatorname{Re}\left(E(z, t) e^{-i \omega_{0} t+i k_{0} z}(\mathbf{x}+i \mathbf{y})\right)
$$

where $E=E_{x}-i E_{y}$ is the slowly varying (with space and time) envelope of the right circularly polarized wave; $\mathbf{x}$ and $\mathbf{y}$ are the unitary vectors along the axes perpendicular to the direction $\mathbf{z}$ of the wave propagation and of the ambient magnetic field $\mathbf{B}_{0}=B_{0} \mathbf{z} ; E_{x}$ and $E_{y}$ are the coordinates of $\mathbf{E}$ along $\mathbf{x}$ and $\mathbf{y} ; \omega_{0}$ and $\mathbf{k}_{0}=k_{0} \mathbf{z}$ are the central frequency and wavenumber of the wave packet. The whistler dispersion relation is given by

$$
\mathcal{D}\left(\omega_{0}, k_{0}\right)=k_{0}^{2} c^{2}-\omega_{0}^{2}+\frac{\omega_{p}^{2} \omega_{0}}{\omega_{0}-\omega_{c}}=0 .
$$

Then the equation of evolution of the wave electric field envelope is obtained in the form ${ }^{42}$

$$
i \frac{\partial E}{\partial t}+i v_{g 0} \frac{\partial E}{\partial z}+\frac{v_{g 0}^{\prime}}{2} \frac{\partial^{2} E}{\partial z^{2}}=-\frac{\omega_{0} \omega_{p}^{2}}{\left(\omega_{0}-\omega_{c}\right) \mathcal{D}_{0}^{\prime}}\left(\rho+\frac{\omega_{c}}{\omega_{0}-\omega_{c}} \frac{k_{0} V}{\omega_{0}}\right) E,
$$

where $\rho=\delta n_{e} / n_{0}$ and $V=\delta v_{e}$ are the perturbations of the slowly varying electron density $n_{e}$ and fluid velocity $v_{e}$, respectively; $n_{0}$ is the unperturbed plasma density at equilibrium, and $\mathcal{D}_{0}^{\prime}=(\partial \mathcal{D} / \partial \omega)_{0}=(\partial \mathcal{D} / \partial \omega)_{k=k_{0}, \omega=\omega_{0}}$ (see also below a similar notation for other variables). The group velocity and its derivative are given by

$v_{g 0}=\left(\frac{d \omega}{d k}\right)_{0}=-\frac{(\partial \mathcal{D} / \partial k)_{0}}{(\partial \mathcal{D} / \partial \omega)_{0}}=-\frac{2 c^{2} k_{0}}{\mathcal{D}_{0}^{\prime}}, \quad v_{g 0}^{\prime}=\left(\frac{d^{2} \omega}{d k^{2}}\right)_{0}=\frac{v_{g 0}}{k_{0}}\left(1-\frac{v_{g 0}^{2}}{c^{2}}\left(1+\frac{\omega_{p}^{2} \omega_{c}}{\left(\omega_{c}-\omega_{0}\right)^{3}}\right)\right)$.

In the case of quasineutral slow oscillations $\left(\rho \simeq \delta n_{e} / n_{0} \simeq \delta n_{i} / n_{0}\right.$, where $\delta n_{i}$ is the ion density perturbation) and, consequently, due to the charge conservation at lowest order ( $V \simeq \delta v_{e} \simeq \delta v_{i}$, where $\delta v_{i}$ is the ion population's velocity perturbation), one can obtain the ion acoustic dynamics in the form ${ }^{42}$

$$
\begin{gathered}
\frac{\partial}{\partial t}\left(V-\frac{k_{0} \omega_{c} \omega_{p}^{2}}{\omega_{0}^{2}\left(\omega_{0}-\omega_{c}\right)^{2}} \frac{|E|^{2}}{16 \pi n_{0} m_{i}}\right)+\frac{\partial}{\partial z}\left(c_{s}^{2} \rho+\frac{\omega_{p}^{2}}{\omega_{0}\left(\omega_{0}-\omega_{c}\right)} \frac{|E|^{2}}{16 \pi n_{0} m_{i}}\right) \simeq 0 \\
\frac{\partial \rho}{\partial t}+\frac{\partial V}{\partial z} \simeq 0,
\end{gathered}
$$


where the hydrodynamic nonlinear terms have been neglected compared to the ponderomotive terms, which include a stationary and a nonstationary part; $c_{s}$ is the ion acoustic velocity and $m_{i}$ is the proton mass. Defining the hydrodynamic flux $\Psi$ in the form

$$
\frac{\partial \Psi}{\partial z}=V-\frac{k_{0} \omega_{c} \omega_{p}^{2}}{\omega_{0}^{2}\left(\omega_{c}-\omega_{0}\right)^{2}} \frac{|E|^{2}}{16 \pi n_{0} m_{i}},
$$

and identifying the couples of canonical variables as $\left(\Psi,-k_{0}^{2} \rho / \mathcal{D}_{0}^{\prime}\right)$ and $\left(C, C^{*}\right)$, with $C=$ $\sigma_{0} E e^{-i \omega_{0} t}$, we get the Hamiltonian of the system without resonant particles as $\mathcal{H}_{w}=$ $\int_{L} H_{w} d z / L$ with

$$
\begin{gathered}
H_{w}=\frac{i v_{g 0}}{2}\left(C \frac{\partial C^{*}}{\partial z}-C^{*} \frac{\partial C}{\partial z}\right)+\frac{v_{g 0}^{\prime}}{2}\left|\frac{\partial C}{\partial z}\right|^{2} \\
+\frac{\omega_{0} \omega_{p}^{2} \rho|C|^{2}}{\left(\omega_{c}-\omega_{0}\right) \mathcal{D}_{0}^{\prime}}+\frac{L n_{0} m_{i}}{2}\left(c_{s}^{2} \rho^{2}+\left(\frac{\partial \Psi}{\partial z}+\frac{k_{0} \omega_{c} \omega_{p}^{2}}{\omega_{0}^{2}\left(\omega_{c}-\omega_{0}\right)^{2}} \frac{|E|^{2}}{16 \pi n_{0} m_{i}}\right)^{2}\right),
\end{gathered}
$$

where we will show below that

$$
\sigma_{0}^{2}=\frac{L \varepsilon_{0}^{\prime}}{16 \pi}
$$

$\varepsilon_{0}^{\prime}=-\mathcal{D}_{0}^{\prime} / \omega_{0}^{2}$ is the derivative of the dielectric constant with respect to frequency, taken at $k=k_{0}$ and $\omega=\omega_{0} ; L$ is the size of the system along $z$. The Hamilton equations are then given by

$$
-\frac{k_{0}^{2}}{\mathcal{D}_{0}^{\prime}} \frac{\partial \rho}{\partial t}=\frac{\delta H_{w}}{\delta \Psi}, \quad \frac{k_{0}^{2}}{\mathcal{D}_{0}^{\prime}} \frac{\partial \Psi}{\partial t}=\frac{\delta H_{w}}{\delta \rho}, \quad \frac{\partial C}{\partial t}=-i \frac{\delta H_{w}}{\delta C^{*}},
$$

where $\delta$ refers to the functional derivative. Note that the choice of the canonical variables $\left(C, C^{*}\right)$ in the form $C=\sigma_{0} E e^{-i \omega_{0} t}$ is due to the fact that they have also to be canonical variables for the full system, i.e. the plasma where a flux of resonant electrons is now introduced. This flux has a density $n_{b}$ which is small compared to that of the background plasma, i.e. $n_{b} \ll n_{0}$. Let us determine the energy conservation law starting from the electrons' motion

$$
\frac{d \mathbf{v}_{p}}{d t}=-\frac{e}{m_{e}} \mathbf{E}-\frac{e}{m_{e} c} \mathbf{v}_{p} \times \mathbf{B}
$$

where $\mathbf{v}_{p}$ is the velocity of the particle $p ; m_{e}$ and $-e<0$ are the electron mass and charge. The components of the electric and magnetic fields $\mathbf{E}$ and $\mathbf{B}$ along the axes $\mathbf{x}$ and $\mathbf{y}$ are given by

$$
E_{x}=\operatorname{Re} \sum_{k} E_{k} e^{-i \omega_{0} t+i\left(k_{0}+k\right) z}, \quad E_{y}=\operatorname{Re} \sum_{k} i E_{k} e^{-i \omega_{0} t+i\left(k_{0}+k\right) z},
$$

where $k \ll k_{0}$, and

$$
B_{x}=-\frac{c k_{0}}{\omega_{0}} E_{y}, \quad B_{y}=\frac{c k_{0}}{\omega_{0}} E_{x}, \quad B_{z}=B_{0}=\frac{\omega_{c} m_{e} c}{e} .
$$


Then we can calculate that

$$
\frac{1}{2} \frac{d \mathbf{v}_{p}^{2}}{d t}=-\frac{e}{m_{e}}\left(v_{p x} E_{x}+v_{p y} E_{y}\right)=-\frac{e}{m_{e}} \operatorname{Re} \sum_{k}\left(v_{p x}+i v_{p y}\right) E_{k} e^{-i \omega_{0} t+i\left(k_{0}+k\right) z}
$$

which allows to write that

$$
\frac{1}{N} \frac{d}{d t} \sum_{p} \frac{m_{e} \mathbf{v}_{p}^{2}}{2}+e \operatorname{Re}\left(\sum_{k} E_{k}^{*} J_{k}\right)=0
$$

with

$$
J_{k}=\frac{1}{N} \sum_{p}\left(v_{p x}-i v_{p y}\right) e^{i \omega_{0} t-i\left(k_{0}+k\right) z_{p}},
$$

where $z_{p}$ is the coordinate of the particle $p$ along $\mathbf{z}$ and $N=L n_{b}$ is the number of macroparticles representing the resonant electrons; $v_{p x}$ and $v_{p y}$ are the components of the velocity of the particle $p$ along $\mathbf{x}$ and $\mathbf{y}$. As in a homogeneous plasma the equation of wave evolution is of the form ${ }^{43}$

$$
\frac{\partial E_{k}}{\partial t}=\alpha J_{k}=\frac{8 \pi e n_{b}}{\omega_{0} \varepsilon_{0}^{\prime}} J_{k}
$$

we get the following energy conservation (17) in the absence of inhomogeneities

$$
\frac{d}{d t}\left(\sum_{p} \frac{m_{e} \mathbf{v}_{p}^{2}}{2}+L \sum_{k} \omega_{0} \varepsilon_{0}^{\prime} \frac{\left|E_{k}\right|^{2}}{16 \pi}\right)=0
$$

The wave energy density is given by

$$
\left(\frac{E_{i}^{*} E_{j}}{16 \pi} \frac{\partial\left(\omega^{2} \hat{\varepsilon}_{i j}^{h}\right)}{\omega \partial \omega}\right)_{0}=\omega_{0} \varepsilon_{0}^{\prime} \frac{|E|^{2}}{16 \pi}
$$

where $\hat{\varepsilon}_{i j}^{h}$ is the Hermitian part of the dielectric tensor $\hat{\varepsilon}_{i j}$ with elements

$$
\varepsilon_{x x}=\varepsilon_{y y}=1-\frac{\omega_{p}^{2}}{\omega^{2}-\omega_{c}^{2}}, \quad \varepsilon_{x y}=-\varepsilon_{y x}=\frac{i \omega_{c} \omega_{p}^{2}}{\omega\left(\omega^{2}-\omega_{c}^{2}\right)} .
$$

Therefore, the total Hamiltonian $H_{t}$ corresponding to the whistlers propagating in the inhomogeneous plasma and interacting with the flux of resonant particles can be written in the form

$$
H_{t}=H_{w}+H_{p}=H_{w}+\sum_{p=1}^{N} h_{p}+\sum_{k} \omega_{0}\left|C_{k}\right|^{2}
$$

where the kinetic energy of a particle $p$ is defined by (with the gauge $\varphi=0$ )

$$
h_{p}=\frac{m_{e} \mathbf{v}_{p}^{2}}{2}=\frac{1}{2 m_{e}}\left(\mathbf{P}_{p}+\frac{e}{c} \mathbf{A}_{0}\left(z_{p}\right)+\frac{e}{c} \operatorname{Re}\left(A\left(z_{p}, t\right) e^{-i \omega_{0} t+i k_{0} z_{p}}(\mathbf{x}+i \mathbf{y})\right)\right)^{2} .
$$


$C_{k}$ is the Fourier component of the canonical variable $C=\sigma_{0} E e^{-i \omega_{0} t} ; \mathbf{P}_{p}$ is the generalized momentum of the particle $p ; \mathbf{A}_{0}$ is the stationary part of the vector potential $\mathbf{A} ; A$ is the envelope of A. The full set of Hamilton equations is then provided by (12) applied to the Hamiltonian $H_{t}(23)$ instead of $H_{w}(10)$

$$
-\frac{k_{0}^{2}}{\mathcal{D}_{0}^{\prime}} \frac{\partial \rho}{\partial t}=\frac{\delta H_{t}}{\delta \Psi}, \quad \frac{k_{0}^{2}}{\mathcal{D}_{0}^{\prime}} \frac{\partial \Psi}{\partial t}=\frac{\delta H_{t}}{\delta \rho}, \quad \frac{\partial C}{\partial t}=-i \frac{\delta H_{t}}{\delta C^{*}},
$$

together with the two additional relations for each particle $p$

$$
\frac{d \mathbf{P}_{p}}{d t}=-\frac{\partial H_{t}}{\partial \mathbf{z}_{p}}, \quad \frac{d \mathbf{z}_{p}}{d t}=\frac{\partial H_{t}}{\partial \mathbf{P}_{p}}
$$

which provide the Newton equations. Using (25) in the Fourier space, the following relation can be derived from the term $H_{p}$ describing the wave-particle interaction in (23)

$$
i \frac{\partial C_{k}}{\partial t}=\frac{\partial}{\delta C_{k}^{*}}\left(\sum_{k} \omega_{0}\left|C_{k}\right|^{2}\right)=\omega_{0} C_{k} .
$$

For the first term $h_{p}$ of $H_{p}(23)$ we can calculate, using the relation $C=\sigma_{0} E e^{-i \omega_{0} t}=$ $i \omega_{0} \sigma_{0} A e^{-i \omega_{0} t} / c$, that

$$
\begin{gathered}
\frac{\delta}{\delta C_{k}^{*}} \sum_{p} h_{p}=\sum_{p} m_{e} \mathbf{v}_{p} \cdot \frac{\delta \mathbf{v}_{p}}{\delta C_{k}^{*}} \\
=\sum_{p} \mathbf{v}_{p} \cdot \frac{\delta}{\delta C_{k}^{*}}\left(\mathbf{P}_{p}+\frac{e}{c} \mathbf{A}_{0}\left(z_{p}\right)+\frac{e}{c} \operatorname{Re}\left(\frac{c}{i \omega_{0} \sigma_{0}} C\left(z_{p}, t\right) e^{i k_{0} z_{p}}(\mathbf{x}+i \mathbf{y})\right)\right),
\end{gathered}
$$

which provides the following expression

$$
i \frac{\partial C_{k}}{\partial t}=\frac{i e}{2 \omega_{0} \sigma_{0}} \sum_{p} \mathbf{v}_{p} \cdot(\mathbf{x}-i \mathbf{y}) e^{-i k z_{p}} e^{-i k_{0} z_{p}} .
$$

Taking into account (27) and (29), we obtain the evolution of the envelope $C$ in the form

$$
i \frac{\partial C}{\partial t}-\omega_{0} C=i e^{-i \omega_{0} t} \frac{\partial}{\partial t}\left(C e^{i \omega_{0} t}\right)=\frac{i e}{2 \omega_{0} \sigma_{0}} \sum_{k} \sum_{p} \mathbf{v}_{p} \cdot(\mathbf{x}-i \mathbf{y}) e^{-i k_{0} z_{p}} e^{-i k z_{p}} e^{i k z}
$$

where summations on all waves and resonant particles are performed in the right hand side term. The parameter $\sigma_{0}$ can be determined by writing that (see (20))

$$
L \sum_{k} \omega_{0} \varepsilon_{0}^{\prime} \frac{\left|E_{k}\right|^{2}}{16 \pi}=\sum_{k} \omega_{0}\left|C_{k}\right|^{2}=\sum_{k} \sigma_{0}^{2} \omega_{0}\left|E_{k}\right|^{2}
$$

which leads to (11), so that (30) can be written as

$$
i \frac{\partial E}{\partial t}=\frac{8 \pi i e}{\varepsilon_{0}^{\prime} \omega_{0}} \frac{n_{b}}{N} \sum_{k} \sum_{p} \mathbf{v}_{p} \cdot(\mathbf{x}-i \mathbf{y}) e^{i \omega_{0} t} e^{-i k_{0} z_{p}} e^{-i k z_{p}} e^{i k z} .
$$


Adding the terms provided by the Hamilton equations for $H_{w}(10)-(12)$, we finally obtain (32) in the form

$i \frac{\partial E}{\partial t}+i v_{g 0} \frac{\partial E}{\partial z}+\frac{v_{g 0}^{\prime}}{2} \frac{\partial^{2} E}{\partial z^{2}}+\frac{\omega_{p}^{2}}{\varepsilon_{0}^{\prime} \omega_{0}\left(\omega_{c}-\omega_{0}\right)} \rho E=-\frac{8 \pi i e \omega_{0}}{\mathcal{D}_{0}^{\prime}} \frac{n_{b}}{N} \sum_{k} \sum_{p}\left(v_{p x}-i v_{p y}\right) e^{-i\left(k+k_{0}\right) z_{p}} e^{i \omega_{0} t} e^{i k z}$

which describes the interaction of the whistlers' electric field envelope with resonant electrons in a plasma with density and velocity inhomogeneities. The right hand side term represents the current contribution of the beam electrons.

The full system to be solved self-consistently consists in the set of equations (7), (8), (13) and (33). A numerical code has been written to solve these equations by using discretization schemes, pseudo-spectral methods and Fast Fourier Transforms. The three-dimensional motion of the individual particles is computed using a leapfrog-type integrator. Owing to the approach used, a limited number $N_{p}$ of resonant particles $\left(N_{p}=100000-500000\right)$ is sufficient to provide clearly interpretable simulation results. Packets of $1024-4096$ waves of wavenumbers $k_{0}+k\left(|k| \ll k_{0}\right)$ and frequencies $\omega_{k_{0}+k}$ are used, which present initially narrowband spectra peaked at the central wavenumber $k_{0}$ at frequency $\omega_{0}$. At the initial state, particles are distributed uniformly in space and described by arbitrary velocity distributions. The one-dimensional simulation box extends along $\mathbf{z}$ over a normalized distance $L \omega_{c} / c \simeq 3000-10000$. More details concerning the numerical scheme are given in the companion paper ${ }^{42}$.

\section{INTERACTION OF WHISTLER ENVELOPE SOLITONS WITH PARTICLES}

In this Section we present results of numerical simulations of whistler envelope solitons interacting with electron fluxes at various conditions typical of the heliospheric plasmas. Note that we present hereafter examples only for a few sets of parameters, but the same qualitative conclusions can be stated for quite different plasma conditions, as shown by our simulations' results. This paper is not devoted to fulfill a parametric analysis but to evidence and explain physical mechanisms. We consider weak and warm electron beams with initial velocity distributions $F_{b}\left(v_{z}, v_{\perp}\right)$, that propagate in the direction opposite to the ambient magnetic field with a velocity $v_{b}$ and a density $n_{b} \ll n_{0}$, and interact with the whistler 
solitons at normal cyclotron resonance velocities $v_{z k}=\left(\omega_{k_{0}+k}-\omega_{c}\right) /\left(k_{0}+k\right)$ distributed within a narrow velocity width $\Delta v$ around the central velocity $v_{z 0}=\left(\omega_{k_{0}}-\omega_{c}\right) / k_{0}$. Initially the wave packet is chosen in the form of a whistler envelope soliton ${ }^{42}$ moving with the group velocity $v_{g 0}$

$$
B(z, t=0)=B_{s} \sec \left(\frac{z-z_{0}}{l_{s}}\right) .
$$

The soliton is located at the initial position $z_{0}$ and propagates along a simulation box of length $L$; its amplitude $B_{s}$ and width $l_{s}$ are related as $l_{s} B_{s}=(1 / \beta)\left(c k_{0} / \omega_{0}\right)$ with $^{42}$

$$
\beta=\left(\frac{1}{16 \pi n_{0} m_{i} c^{2}} \frac{\omega_{p}^{4}}{\left(\omega_{0}-\omega_{c}\right)^{2}} \frac{v_{g 0}}{2 k_{0} v_{g 0}^{\prime}}\left(1+\frac{\omega_{c}}{\omega_{0}-\omega_{c}} \frac{k_{0} v_{g 0}}{\omega_{0}}\right)^{2} \frac{1}{\left(c_{s}^{2}-v_{g 0}^{2}\right)}\right)^{1 / 2} .
$$

The corresponding density and fluid velocity perturbations ${ }^{42}$, i.e. $\rho(z, t=0)=\rho_{0} \sec ^{2}\left(\left(z-z_{0}\right) / l_{s}\right)$ and $V(z, t=0)=V_{0} \sec ^{2}\left(\left(z-z_{0}\right) / l_{s}\right)$, are moving with the same velocity as the field envelope. Note that in (35) the ratio $v_{g 0} / k_{0}$ is always positive, as well as the term $v_{g 0}^{\prime}\left(c_{s}^{2}-v_{g 0}^{2}\right)$; the soliton is either supersonic $\left(v_{g 0}>c_{s}\right)$ when $v_{g 0}^{\prime}<0$, either subsonic $\left(v_{g 0}<c_{s}\right)$ when $v_{g 0}^{\prime}>0$.

Figure 2 shows the damping of a high intensity whistler soliton when it interacts with the tail of a Maxwellian electron velocity distribution centered at $v_{z}=0$. The slow decrease with time of the energy density variation of the soliton, i.e. $\Delta W=W(t)-W(0)$ (Fig. 2a), shows that it keeps most of its energy during several tens of thousands of cyclotron periods $\omega_{c}^{-1}$; eventually the soliton has lost only a few percents of its energy $(\Delta W / W(0) \simeq 2 \%$ at $\omega_{c} t \simeq 35000$ ). During its propagation the magnetic field envelope (34) is accompanied by the slow fluid velocity and density perturbations $V$ and $\rho$ which move with the same velocity (see Fig. 3). The profile of the magnetic field envelope is only very slightly deformed (at the bottom edge of the upstream front) after its travel along the whole box. Meanwhile resonant particles are accelerated, as shown by the time evolution of the variations of their parallel, perpendicular and total kinetic energy densities, i.e. $\Delta K_{z}=K_{z}(t)-K_{z}(0)<0$, $\Delta K_{\perp}=K_{\perp}(t)-K_{\perp}(0)>0$ and $\Delta K_{t}=K_{t}(t)-K_{t}(0)>0$, respectively (Fig. $2 \mathrm{~b}$ ). The parallel kinetic energy of the particles is decreased whereas the perpendicular and the total ones are increased. Moreover, $\Delta K_{t}$ is shown on Fig. 2a together with $\Delta W$ as well as the sum $\Delta K_{t}+\Delta W \simeq 0$ (dashed line) demonstrating the total energy's conservation.

As the size of the simulation box is limited and the boundary conditions are periodic, one has to take care in the frame of this study that all electrons should only travel one time 


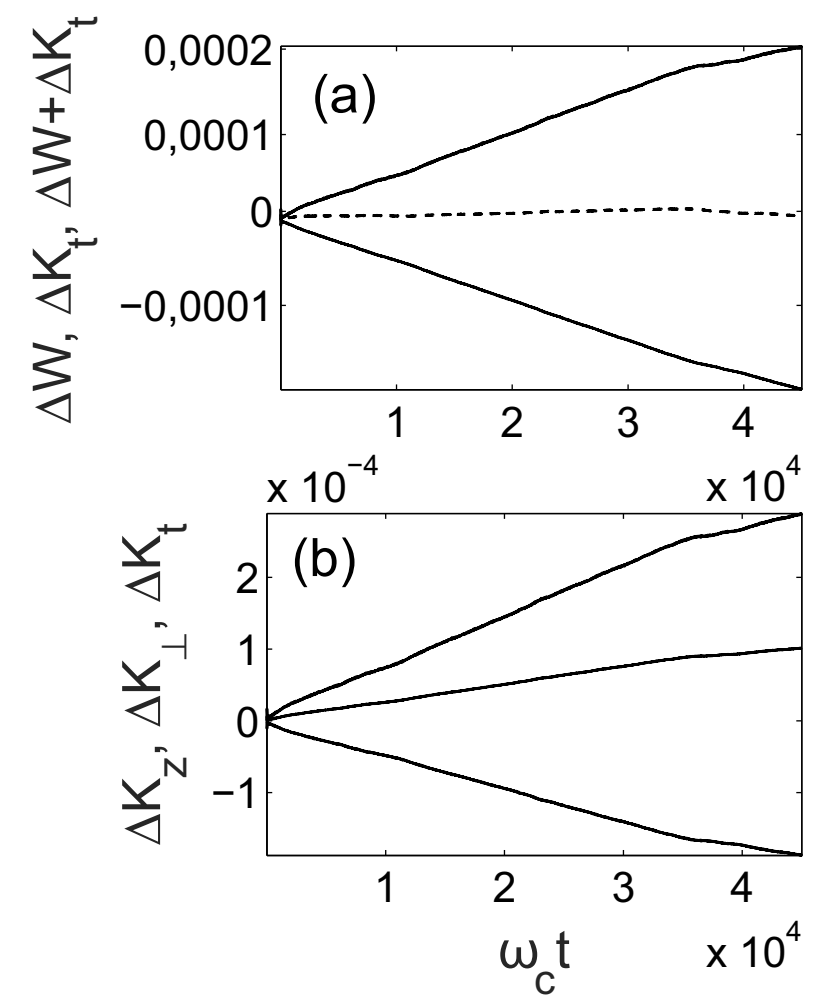

FIG. 2. Soliton interacting with a Maxwellian tail. (a) Time evolution of the variations of the soliton energy density $\Delta W=W(t)-W(0)$ (lower curve) and of the total kinetic energy of the resonant electrons, $\Delta K_{t}=K_{t}(t)-K_{t}(0)$ (upper curve), in arbitrary units; the dashed curve represents the vanishing total energy variation $\Delta W+\Delta K_{t}=0$. (b) Time evolution of the variations of the parallel (lower curve), the perpendicular (upper curve) and the total (middle curve) kinetic energy densities $\Delta K_{z}, \Delta K_{\perp}$ and $\Delta K_{t}$ of the resonant electrons, in arbitrary units. Main parameters are $: L c / \omega_{c}=3500, v_{T} / c=0.005, \omega_{p} / \omega_{c}=17, \omega_{0} / \omega_{c} \simeq 0.35, c k_{0} / \omega_{c}=12.6, n_{b} / n_{0}=0.00002$, $-12.5 v_{T} \lesssim v_{z k} \lesssim-9.5 v_{T}$

across the soliton. Therefore the parameters have been chosen so that the solitons have the time to travel along roughly the full length of the box before they cross the counterstreaming particles for a second time. When this moment is reached the simulation has to be stopped. In order to understand how the soliton interacts with the particles, we examined the time evolution of the resonant electrons' velocity distributions in the plane $v_{z}-v_{\perp}$ for three different populations : (i) the first one corresponds to the particles located inside the soliton region, (ii) the second one to those moving upstream the soliton (right part of the simulation 

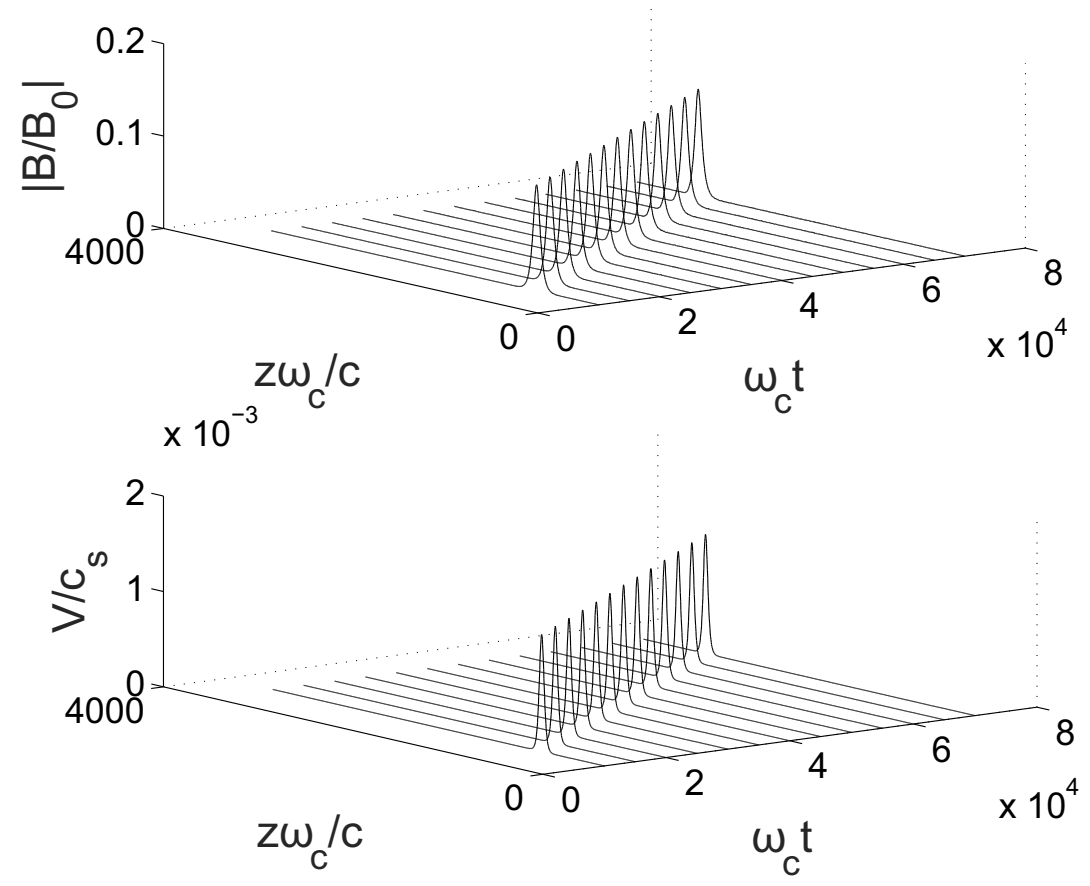

FIG. 3. Whistler envelope soliton along the simulation box at different times. (Upper panel) : Magnetic field envelope $B / B_{0}$. (Lower panel) : Fluid velocity perturbation $V / c_{s}$. Parameters are the same as in Fig. 2.

box) and (iii) the third one to those moving downstream it (left part of the box, where the soliton is initially located). Note that the particles move with a negative parallel velocity and the soliton with a positive one so that, at a given time, those located in the downstream (resp. upstream) region have already interacted (resp. did not already interact) with the soliton; moreover, the upstream population can contain some electrons that have been reinjected at the right boundary of the box (due to the periodic boundary conditions) and are originating from the downstream region. The beam velocity distributions $F_{b}\left(v_{z}, v_{\perp}\right)$ are represented in Fig. 4 for each population (columns) and for 3 time moments (rows). The first statement provided by this analysis is that the soliton interacts locally with the particles; indeed, one observes that the distributions upstream and downstream the soliton are only weakly affected by their interactions with the nonlinear waves, contrary to those of the electron population located in the soliton region, i.e. of particles with positions $z_{p}(t)$ satisfying the condition $\left|z_{p}(t)-z_{0 s}(t)\right| \lesssim l_{s}$, where $z_{0 s}(t)$ is the coordinate of the center of the soliton at time $t$. One 

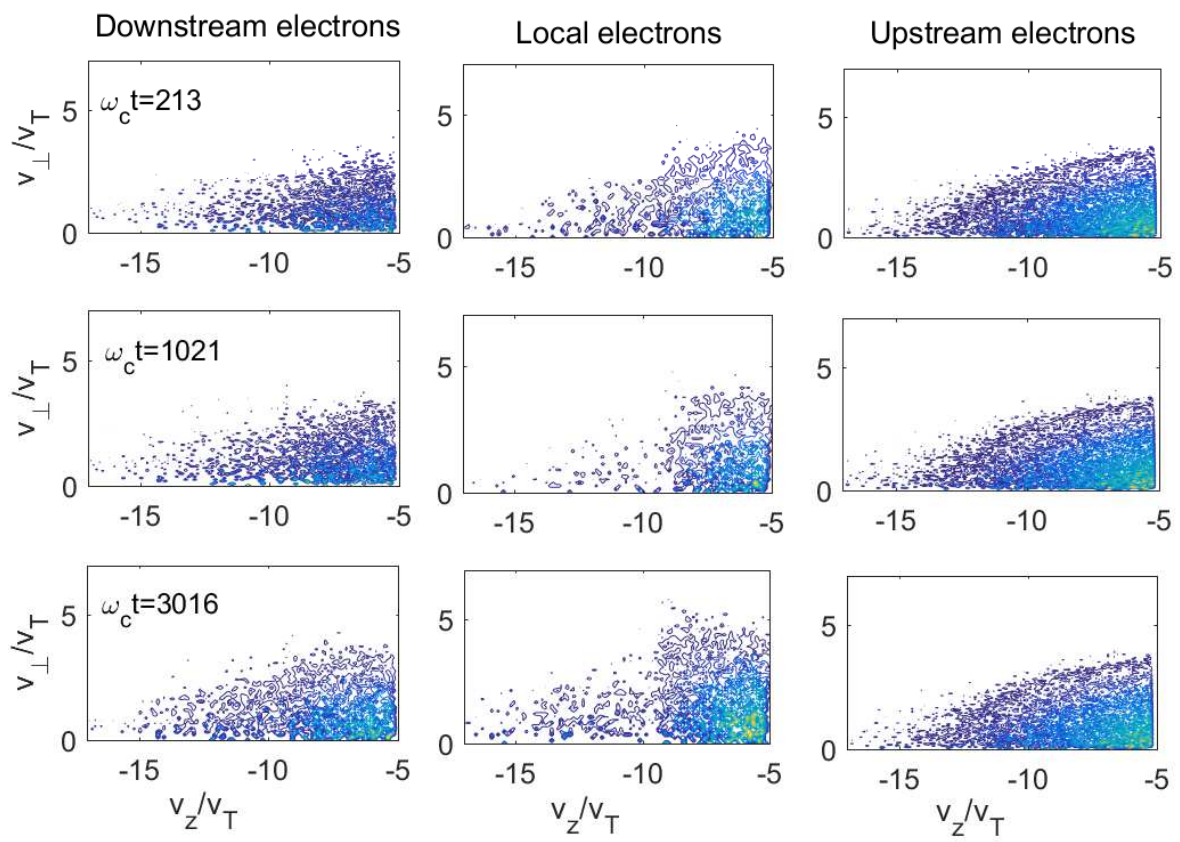

FIG. 4. (Color online) Velocity distributions in the plane $v_{z}-v_{\perp}$ at the times $\omega_{c} t=213$ (upper row), 1021 (middle row), and 3016 (bottom row), for the three electron populations described in the text. (Left column) : downstream electrons; (Middle column) : local electrons; (Right column): upstream electrons. Velocities are normalized by the electron plasma thermal velocity $v_{T}$. Parameters are the same as in Fig. 2.
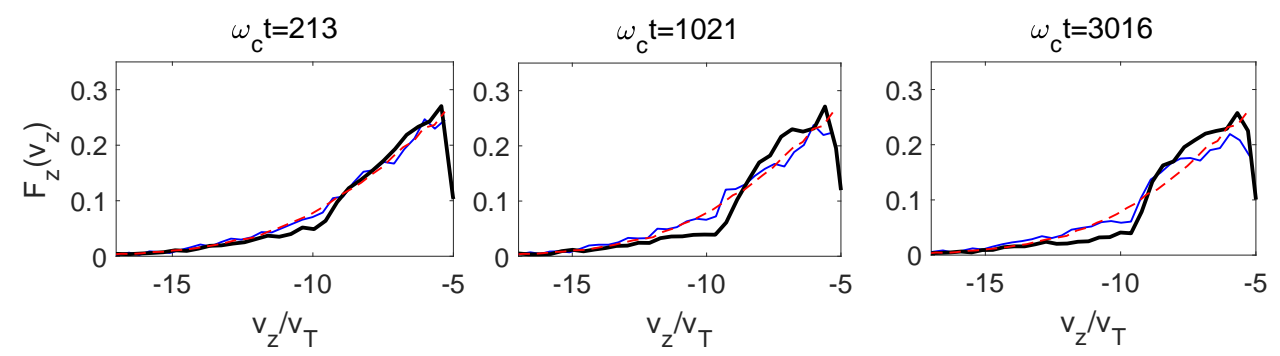

FIG. 5. (Color online) Parallel velocity distributions $F_{z}\left(v_{z}\right)$ corresponding to the three electron populations of Fig. 4, at the same three time moments $\omega_{c} t=213,1021$, and 3016. For each panel : local electrons (thick black lines), downstream electrons (dashed lines - red dashed online), upstream electrons (thin black lines - blue lines online). Parameters are the same as in Fig. 2. 


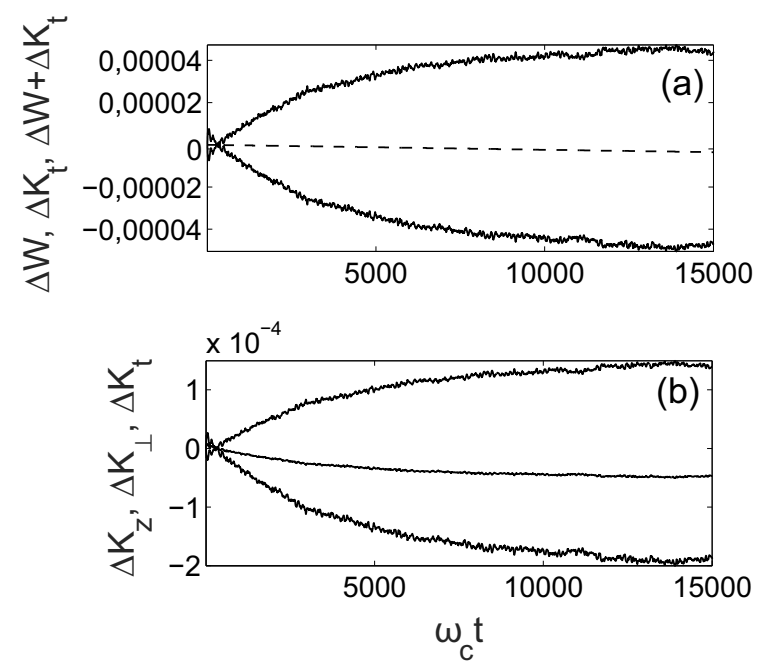

FIG. 6. Soliton interaction with an electron beam. (a) Time evolution of the variations of the soliton energy density $\Delta W=W(t)-W(0)$ (upper curve) and of the total kinetic energy of the resonant electrons, $\Delta K_{t}=K_{t}(t)-K_{t}(0)$ (lower curve), in arbitrary units; the dashed curve represents the vanishing total energy variation $\Delta W+\Delta K_{t}=0$. (b) Time evolution of the variations of the parallel (upper curve), the perpendicular (lower curve) and the total (middle curve) kinetic energy densities $\Delta K_{z}, \Delta K_{\perp}$ and $\Delta K_{t}$ of the resonant electrons, in arbitrary units. Main parameters are $: L c / \omega_{c}=7000, v_{T} / c=0.006, \omega_{p} / \omega_{c}=10, \omega_{0} / \omega_{c} \simeq 0.25, c k_{0} / \omega_{c}=5.8, n_{b} / n_{0}=0.00005$, $v_{b} / v_{T}=-17$.

can clearly see in Fig. 4 that at times $\omega_{c} t \simeq 1021$ and $\omega_{c} t \simeq 3016$ particles have escaped from the region $-12.5 v_{T} \lesssim v_{z k} \lesssim-9.5 v_{T}$ of resonant velocities $v_{z k}=\left(\omega_{k_{0}+k}-\omega_{c}\right) /\left(k_{0}+k\right)$; they have decelerated to velocities $\left|v_{z k}\right| \lesssim 9.5 v_{T}$, as evidenced by the parallel velocity distribution presented in Fig. 5.

If the interactions of the resonant electrons with the solitary structure occur mainly locally, the soliton is however responsible for the perturbation of the particles' distribution in its downstream region which is modified by its passage, what is not expectable a priori. Note that the position of the soliton is not connected with the resonance conditions (depending on the velocities) that govern its interactions with the beam. The condition on locality is not a sufficient condition for efficient interactions between the solitary structure and the particles.

A second example shows the interaction of a whistler soliton of weak intensity with an 
electron velocity distribution in conditions when the soliton can absorb energy from the beam particles, as shown by the evolution of the energy density variation $\Delta W$ (Fig. 6a). The energy growth occurs up to $\omega_{c} t_{s} \simeq 15000$ where stabilization occurs. The electrons are accelerated along the magnetic field $\left(\Delta K_{z}>0\right)$ whereas the perpendicular $\Delta K_{\perp}$ and the total $\Delta K_{t}$ kinetic energy density's variations decrease with time (Fig. 6b). The balance of energy $\Delta K_{t}+\Delta W=0$ is represented by the dashed curve.

The dynamics of the interaction of a whistler soliton with a particle distribution in a inhomogeneous plasma characterized by a density well of the order of $1 \%$ of the average plasma density and a scale significantly larger than the soliton width has been studied. One can observe in Fig. 7a that the soliton damps much more quickly than in the case of Fig. 2a; it has lost almost half of its energy at time $\omega_{c} t \simeq 35000$ and, consequently, a significative increase of kinetic energy (acceleration of particles) has occurred ( $\Delta K_{t}>0$ in Fig. $7 \mathrm{~b}$, with $\left.\Delta K_{\perp}>0, \Delta K_{z}<0\right)$. Meanwhile the shape of the soliton experiences a strong modification (Fig. 8) : the upstream front has steepened whereas oscillations have appeared in the downstream region. The dissipation encountered by the soliton is strong enough to lead to such structure. Moreover, we present in Fig. 9 the same kind of picture as in Fig. 4 , i.e. the time evolution in the plane $v_{z}-v_{\perp}$ of the velocity distributions of the three electron populations (downstream, local and upstream). The conclusion is similar to that stated previously : the soliton interacts mainly with the particles located in its vicinity at a given time, the others participating only weakly. The same simulation has been performed without the particles, but keeping the plasma inhomogeneity. All effects observed above are no more visible : they are actually generated by the interactions with electrons and not by the density inhomogeneity.

For comparison we present the case of a soliton traveling in an inhomogeneous plasma with a density well but interacting with another distribution of particles. The velocity distributions at three different times for the three electron populations mentioned above (see Fig. 10) show that significative particle deceleration and perpendicular heating affect the local population and perturb slightly the downstream one (see the middle and left columns of Fig. 10). Meanwhile the soliton damps and has lost around $5 \%$ of its energy at time $\omega_{c} t \simeq 40000$, i.e. much less than in the previous case. In spite of this small loss of energy, the shape of the soliton is modified as the front has slightly steepened. Note that a simulation performed with the same parameters but with a homogeneous plasma provides 

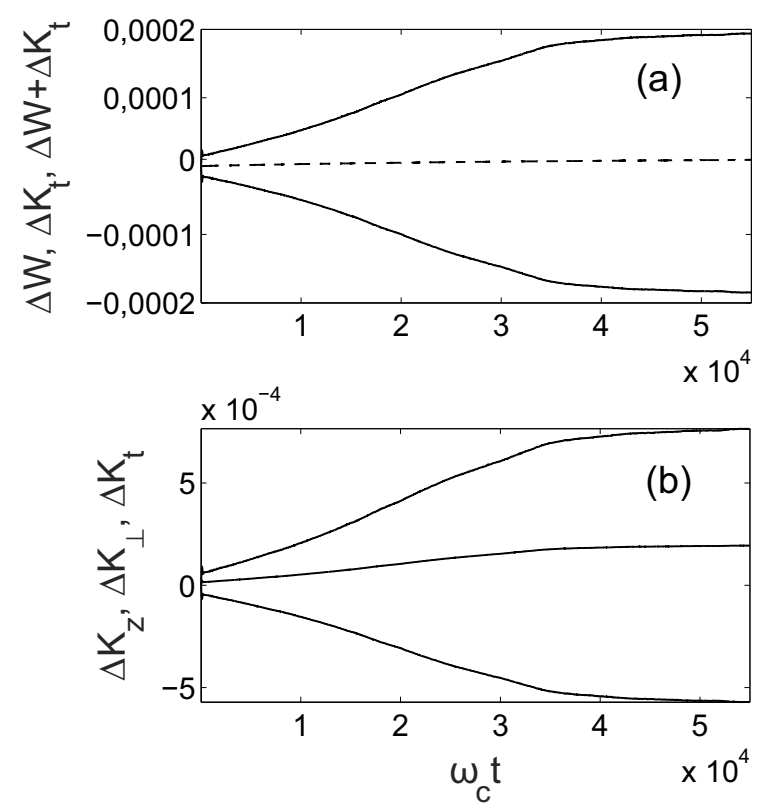

FIG. 7. Soliton interaction with an electron beam in a inhomogeneous plasma. (a) Time evolution of the variations of the soliton energy density $\Delta W$ (lower curve) and of the total kinetic energy of the resonant electrons, $\Delta K_{t}$ (upper curve), in arbitrary units; the dashed curve represents the vanishing total energy variation $\Delta W+\Delta K_{t}=0$. (b) Time evolution of the variations of the parallel (lower curve), the perpendicular (upper curve) and the total (middle curve) kinetic energy densities $\Delta K_{z}, \Delta K_{\perp}$ and $\Delta K_{t}$ of the resonant electrons, in arbitrary units. Main parameters are $: L c / \omega_{c}=7000, v_{T} / c=0.006, \omega_{p} / \omega_{c}=10, \omega_{0} / \omega_{c} \simeq 0.25, c k_{0} / \omega_{c}=5.8, n_{b} / n_{0}=0.00005$, $v_{b} / v_{T}=-25$.

the same results, showing that, as expected, the large scale inhomogeneity does not play a significant role, even if the soliton experiences very small acceleration and deceleration when it travels through it in absence of particles ${ }^{42}$.

On another hand, the rate of exchange of energy (loss or gain) between a whistler soliton and an electron beam can also be estimated analytically in the frame of the electromagnetic quasilinear theory of the weak turbulence. However this approach has some limitations, due partly to the fact that it can not take into account the local character of the interaction evidenced above. Nevertheless we can determine by such calculation under what physical conditions an envelope soliton of small or moderate intensity can loose or gain energy during its interactions with the particles and quantify these exchanges. Moreover the estimation 


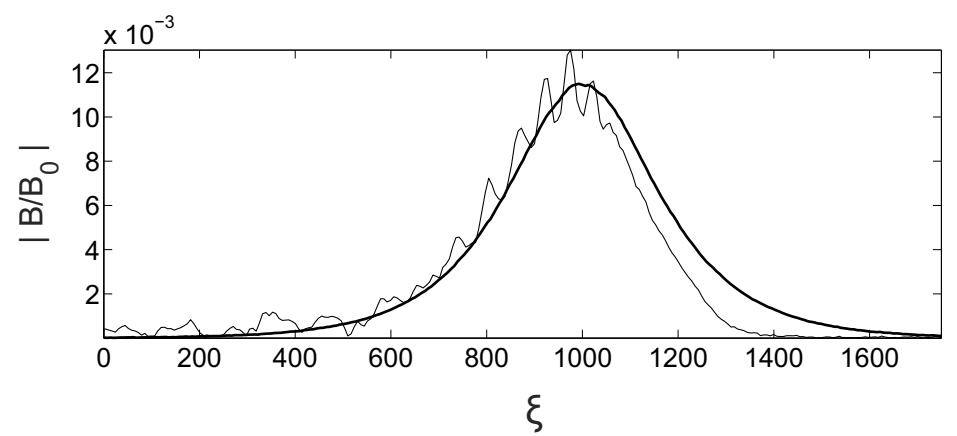

FIG. 8. Profile of the magnetic field envelope soliton $B / B_{0}$ as a function of the coordinate $\xi=$ $z-v_{g 0} t$ in the frame moving with the soliton velocity $v_{g 0}$, at times $\omega_{c} t=10500$ (thin line) and $\omega_{c} t=0$ (thick line). Parameters are the same as in Fig. 7.
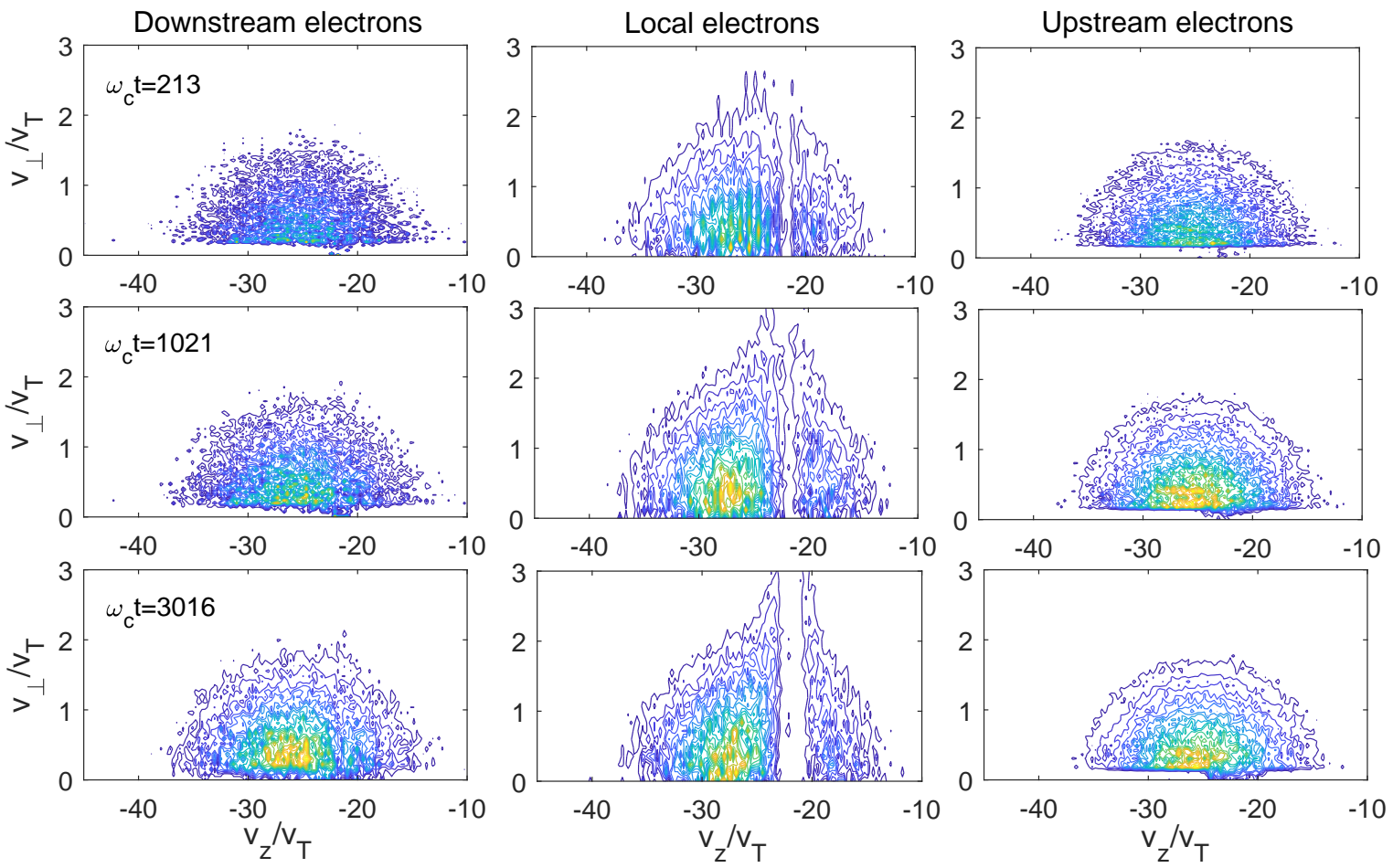

FIG. 9. (Color online) Velocity distributions in the plane $v_{z}-v_{\perp}$ at the times $\omega_{c} t=213$ (upper row), 1021 (middle row), and 3016 (bottom row), for the three electron populations described in the text. (Left column) : downstream electrons; (Middle column) : local electrons; (Right column): upstream electrons. Parameters are the same as in Fig. 7. 
Downstream electrons
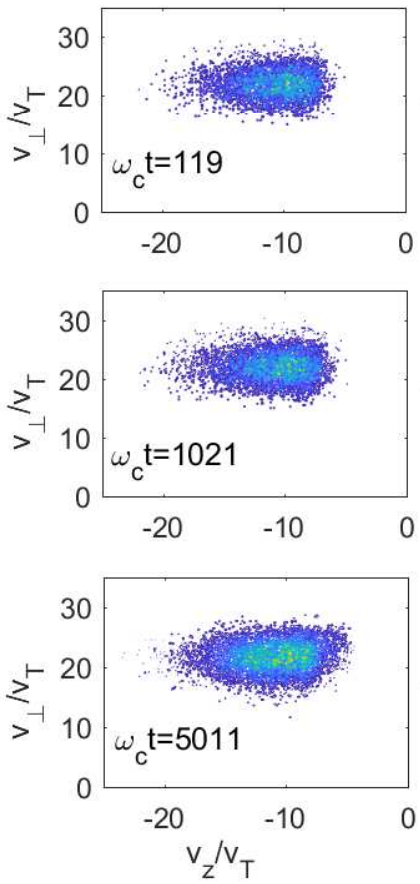

Local electrons
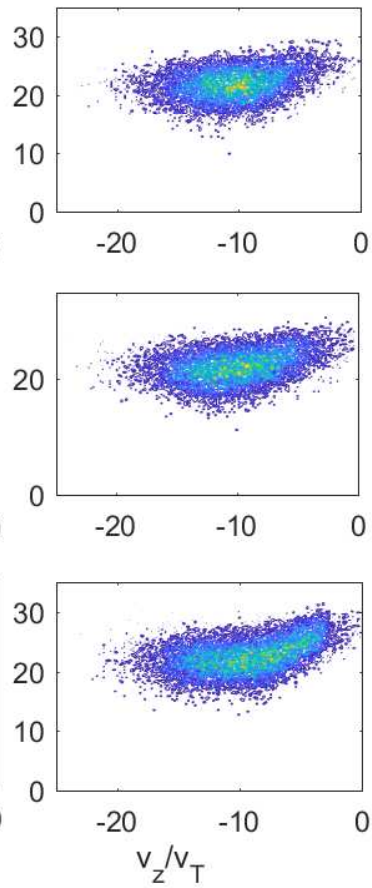

Upstream electrons
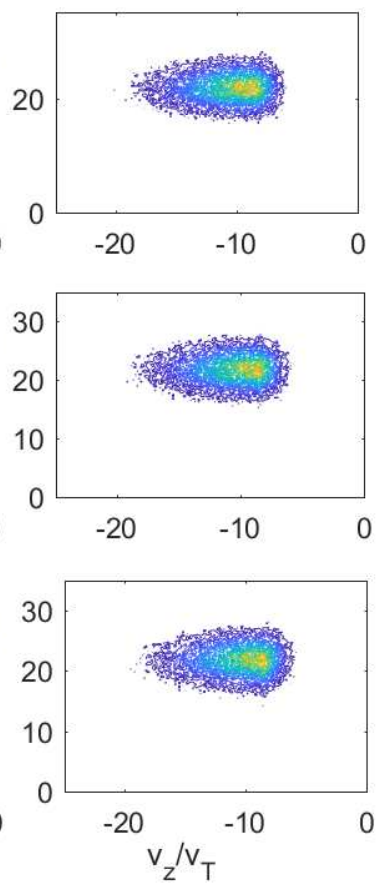

FIG. 10. (Color online) Velocity distributions in the plane $v_{z}-v_{\perp}$ at the times $\omega_{c} t=119$ (upper row), 1021 (middle row), and 5011 (bottom row), for the three electron populations described in the text. (Left column) : downstream electrons; (Middle column) : local electrons; (Right column): upstream electrons. Main parameters are : $L c / \omega_{c}=3500, v_{T} / c=0.005, \omega_{p} / \omega_{c}=17, \omega_{0} / \omega_{c} \simeq 0.4$, $c k_{0} / \omega_{c}=14.4, n_{b} / n_{0}=0.00002, v_{b} / v_{T}=-9$.

given below on the basis of such analytical study shows a rather good agreement with the numerical simulations' results based on the Hamiltonian model. Another more complex approach, which consists in calculating the rate of energy exchange of a moving envelope soliton only with those particles located in its vicinity at a give time, is currently under investigation and will be the subject of a forthcoming paper.

In the frame of electromagnetic quasilinear theory, the slow time variation of the spaceaveraged electron distribution function $F_{b}\left(v_{z}, v_{\perp}, t\right)$ can be written in the form ${ }^{44}$

$$
\begin{gathered}
\frac{\partial F_{b}\left(v_{z}, v_{\perp}, t\right)}{\partial t}=\frac{\pi e^{2}}{m_{e}^{2}} \sum_{k}\left|E_{k}\right|^{2} \times \\
\times\left(\frac{k v_{\perp}}{\omega_{k}} \frac{\partial}{\partial v_{z}}+\left(1-\frac{k v_{z}}{\omega_{k}}\right) \frac{1}{v_{\perp}} \frac{\partial}{\partial v_{\perp}} v_{\perp}\right) \delta\left(\omega_{k}-k v_{z}-\omega_{c}\right)\left(\frac{k v_{\perp}}{\omega_{k}} \frac{\partial}{\partial v_{z}}+\left(1-\frac{k v_{z}}{\omega_{k}}\right) \frac{\partial}{\partial v_{\perp}}\right) F_{b}\left(v_{z}, v_{\perp}, t\right),
\end{gathered}
$$


where $\delta$ is the Dirac function. Note that $\left|E_{k}\right|^{2}$ in Eqs. (36) and (14) have different dimensions; here $\left|E_{k}\right|^{2}$ is an energy density per surface whereas in Eq. (14) it is an energy density per volume.Thus the time variation of the average kinetic energy density $W_{K}$ of the resonant electrons (of density $n_{b}$ ) is given by

$$
\begin{gathered}
\frac{\partial W_{K}}{\partial t}=\int_{0}^{\infty} \int_{-\infty}^{\infty} \frac{m_{e} n_{b} v^{2}}{2} \frac{\partial F_{b}}{\partial t} d \mathbf{v}=\frac{\pi e^{2} n_{b}}{2 m_{e}} \sum_{k}\left|E_{k}\right|^{2} \times \\
\times \int_{0}^{\infty} \int_{-\infty}^{\infty}\left(\frac{k v_{\perp}}{\omega_{k}} \frac{\partial}{\partial v_{z}}+\left(1-\frac{k v_{z}}{\omega_{k}}\right) \frac{1}{v_{\perp}} \frac{\partial}{\partial v_{\perp}} v_{\perp}\right)\left(\delta\left(\omega_{k}-k v_{z}-\omega_{c}\right) G\left(v_{z}, v_{\perp}, t\right)\right)\left(v_{\perp}^{2}+v_{z}^{2}\right) d \mathbf{v}
\end{gathered}
$$

where $d \mathbf{v}=2 \pi v_{\perp} d v_{z} d v_{\perp}$ and

$$
G\left(v_{z}, v_{\perp}, t\right)=\left(\frac{k v_{\perp}}{\omega_{k}} \frac{\partial}{\partial v_{z}}+\left(1-\frac{k v_{z}}{\omega_{k}}\right) \frac{\partial}{\partial v_{\perp}}\right) F_{b}\left(v_{z}, v_{\perp}, t\right) .
$$

By integrating (37) on $v_{z}$ we get

$$
\begin{gathered}
\frac{\partial W_{K}}{\partial t}=\frac{\pi}{4} \omega_{p}^{2} \frac{n_{b}}{n_{0}} \sum_{k} \frac{\left|E_{k}\right|^{2}}{k} \int_{0}^{\infty}\left[v_{z 0}^{2} \frac{k v_{\perp}^{2}}{\omega_{k}}\left(\frac{\partial G}{\partial v_{z}}\right)_{v_{z 0}}\right. \\
\left.+\left(v_{\perp}^{2}+v_{z 0}^{2}\right)\left(1-\frac{k v_{z 0}}{\omega_{k}}\right)\left(\frac{\partial\left(v_{\perp} G\right)}{\partial v_{\perp}}\right)_{v_{z 0}}-\frac{k v_{\perp}^{2}}{\omega_{k}}\left(\frac{\partial}{\partial v_{z}}\left(G v_{z}^{2}\right)\right)_{v_{z 0}}\right] d v_{\perp},
\end{gathered}
$$

where $v_{z 0}=\left(\omega_{k}-\omega_{c}\right) / k<0$ is the normal cyclotron resonant velocity of the wave $\left(\omega_{k}, k\right)$. Let us suppose that initially the resonant particles' velocity distribution $F_{b}\left(v_{z}, v_{\perp}, t=0\right)$ is a beam propagating along $z$ in the direction opposite to the ambient magnetic field with an arbitrary perpendicular distribution $F_{\perp}\left(v_{\perp}\right)$

$$
F_{b}\left(v_{z}, v_{\perp}, t=0\right)=F_{z}\left(v_{z}-v_{b}\right) F_{\perp}\left(v_{\perp}\right),
$$

where $v_{b}<0$ is the beam velocity. The normalization is done according to $2 \pi \int v_{\perp} F_{\perp}\left(v_{\perp}\right) d v_{\perp}=$ 1 and the mean perpendicular velocity square is given by

$$
\left\langle v_{\perp}^{2}\right\rangle=2 \pi \int_{0}^{\infty} v_{\perp}^{3} F_{\perp}\left(v_{\perp}\right) d v_{\perp} .
$$

Performing further integrations on $v_{\perp}$ in (39), we get the time variation of the density of kinetic energy in the form

$$
\frac{\partial W_{K}}{\partial t} \simeq \frac{\omega_{p}^{2}}{8} \frac{n_{b}}{n_{0}} \sum_{k}\left|E_{k}\right|^{2} \frac{\mathcal{F}_{k}}{k}
$$


where

$$
\mathcal{F}_{k}=\frac{k^{2}\left\langle v_{\perp}^{2}\right\rangle}{\omega_{k}^{2}}\left(v_{z 0}^{2} F_{z}^{\prime \prime}\left(v_{z 0}\right)-2 v_{z 0} F_{z}^{\prime}\left(v_{z 0}\right)\right)+4\left(1-\frac{k v_{z 0}}{\omega_{k}}\right) F_{z}\left(v_{z 0}\right) .
$$

$F_{z}^{\prime}\left(v_{z 0}\right)$ and $F_{z}^{\prime \prime}\left(v_{z 0}\right)$ are the first and the second order derivatives of $F_{z}\left(v_{z}\right)$ with respect to $v_{z}$, at $v_{z}=v_{z 0}$. As for the system of waves interacting with the resonant particles the total energy is conserved in the considered plasma of size $L$, we can write for the wave $\left(\omega_{0}, k_{0}\right)$ that

$$
-L \frac{\partial}{\partial t} W_{K}=\frac{\partial W_{p}}{\partial t}=\left(1+\frac{c^{2} k_{0}^{2}}{\omega_{0}^{2}}+\frac{\omega_{p}^{2}}{\left(\omega_{0}-\omega_{c}\right)^{2}}\right) \frac{\partial}{\partial t} \int \frac{|E|^{2}}{4 \pi} d \xi,
$$

where the energy $W_{p}$ carried by the waves has been derived from (21) and $\xi=z-v_{g 0} t$. Thus the rate of change of the energy $W_{\text {sol }}$ of a single soliton inside the box of size $L$ is

$$
\frac{\partial}{\partial t} \int \frac{|E|^{2}}{4 \pi} d \xi=\frac{\partial}{\partial t} W_{s o l}=-\frac{\omega_{p}^{2}}{8} \frac{n_{b}}{n_{0}}\left(1+\frac{c^{2} k_{0}^{2}}{\omega_{0}^{2}}+\frac{\omega_{p}^{2}}{\left(\omega_{0}-\omega_{c}\right)^{2}}\right)^{-1} L \sum_{k}\left|E_{k}\right|^{2} \frac{\mathcal{F}_{k}}{k} .
$$

In the frame moving with the whistler group velocity $v_{g 0}$, the electric field of the whistler envelope soliton satisfies $E^{2}(\xi, t)=E_{s}^{2} \sec ^{2}\left(\xi / l_{s}\right)$. The energy carried by a soliton of size $l_{s} \ll L$ is

$$
W_{s o l}=\int \frac{|E|^{2}}{4 \pi} d \xi \simeq \frac{\left|E_{s}\right|^{2} l_{s}}{2 \pi},
$$

where $\beta^{-1}=E_{s} l_{s}(35)$. Then for the single soliton we can write that

$$
E_{k}=\frac{1}{L} \int_{0}^{L} e^{-i k \xi} E d \xi=E_{s} \frac{\pi l_{s}}{L} \sec \left(\frac{\pi\left(k-k_{0}\right) l_{s}}{2}\right),
$$

where we took into account the form of the electric field packet (3). Considering the quasimonochromatic character of the wave packet, we get

$$
\left|E_{k}\right|^{2}=\frac{E_{s}^{2} \pi^{2} l_{s}^{2}}{L^{2}} \sec ^{2}\left(\frac{\pi\left(k-k_{0}\right) l_{s}}{2}\right) \simeq \frac{\pi^{2} E_{s}^{2} l_{s}^{2}}{L^{2}} \delta_{k k_{0}}
$$

where the discrete Dirac function $\delta_{k k_{0}}$ satisfies $\delta_{k k_{0}}=1$ if $k=k_{0}$ and $\delta_{k k_{0}}=0$ otherwise. Thus, after summation on the wavenumber $k$ in (45), the rate of energy variation for one soliton inside the box is

$$
\frac{\partial W_{\text {sol }}}{\omega_{c} \partial t}=\frac{\gamma}{\omega_{c}} W_{\text {sol }},
$$

with the normalized growth/damping rate

$$
\frac{\gamma}{\omega_{c}} \simeq-\frac{\pi^{3}}{4} \frac{\omega_{p}^{2}}{\omega_{c}} \frac{n_{b}}{n_{0}}\left(1+\frac{c^{2} k_{0}^{2}}{\omega_{0}^{2}}+\frac{\omega_{p}^{2}}{\left(\omega_{0}-\omega_{c}\right)^{2}}\right)^{-1} \frac{\mathcal{F}_{k_{0}}}{k_{0}} \frac{l_{s}}{L} .
$$


The sign of the growth rate $\gamma$ depends only on the sign of $\mathcal{F}_{k_{0}}(43)$. In the limit when the whistler frequency $\omega_{0}$ is very low, i.e. when $\omega_{0} \ll \omega_{c}<\omega_{p}$ and the dispersion relation is thus $\omega_{0} \simeq c^{2} k_{0}^{2} \omega_{c} / \omega_{p}^{2}$, we obtain

$$
\frac{\gamma}{\omega_{c}} \simeq-\frac{\pi^{3}}{4} \frac{\omega_{c}}{k_{0}} \frac{n_{b}}{n_{0}}\left(\frac{\omega_{c}}{\omega_{0}}\left\langle v_{\perp}^{2}\right\rangle\left(F_{z}^{\prime \prime}\left(v_{z 0}\right)+\frac{2 k_{0}}{\omega_{c}} F_{z}^{\prime}\left(v_{z 0}\right)\right)+4 F_{z}\left(v_{z 0}\right)\right) \frac{l_{s}}{L}
$$

Let us now consider $N$ solitons distributed within the whole box of length $L$. Their resultant energy is $W_{s}=\sum N\left(l_{s}, k_{0}\right) W_{\text {sol }}\left(l_{s}, k_{0}\right) / N$, where $N\left(l_{s}, k_{0}\right)$ is the number of solitons of width $l_{s}$ and central wavenumber $k_{0}$, and $W_{\text {sol }}\left(l_{s}, k_{0}\right)$ is the energy of such a soliton (48). If the solitons' positions and phases are not correlated, the rate of the soliton energy density variation is given by

$$
\frac{\partial W_{s}}{\omega_{c} \partial t}=\sum_{l_{s}, k_{0}} \frac{N\left(l_{s}, k_{0}\right)}{N} \frac{\gamma\left(l_{s}, k_{0}\right)}{\omega_{c}} W_{s o l}\left(l_{s}, k_{0}\right)
$$

To use this formula one needs to know the distribution of the solitons' characteristics as a function of the scales $l_{s}$ (or amplitudes $E_{s}$ ) and the wavenumbers $k_{0}$. Unfortunately it is not the case. Nevertheless, let us compare the rate of energy variation of one soliton within the box of length $L$, obtained owing to the numerical simulations, with the value of $\gamma / \omega_{c}$ calculated according to (49). Figure 11 shows the variation of $\gamma / \omega_{c}(49)$ with the resonant velocity $v_{z 0}=\left(\omega-\omega_{c}\right) / k$ for the parameters of Fig. 2 (upper panel) and Fig. 6 (lower panel). For the former (latter) case the soliton has lost around 4\% (has gained around $8 \%$ ) of its energy at the time $\omega_{c} t_{1}=35000$. The initial resonant velocity domain of the soliton is located, for the parallel velocity distribution of Fig. 2 (Fig. 6), in the region $-12 \lesssim v_{z 0} \lesssim-9$ where $\gamma / \omega_{c}<0\left(-22.5 \lesssim v_{z 0} \lesssim-19.5\right.$ where $\gamma / \omega_{c}>0$ for $\left.-21.5 \lesssim v_{z 0}\right)$. Estimates calculated using (48)-(49) are in good agreement with the numerical simulations' results as one obtains in the first case, for $N=1$ and $l_{s} / L \simeq 1 / 30$, a relative loss of energy of $4 \%$ and, in the second case, for $N=1$ and $l_{s} / L \simeq 1 / 40$, a relative gain of energy of $19 \%$.

Finally, let us mention that numerical simulations performed in the frame of this study (but not presented here because the interactions between the solitons and the particles are not efficient) have shown that whistler solitons can keep their shape and their stability during time periods reaching up to $10^{6}-10^{8} \omega_{c}^{-1}$ and even more, depending on the physical parameters, what corresponds to growth/damping rates $\gamma / \omega_{c}$ significantly smaller than those presented in Figs. 11a-b. Moreover, the occurrence during their travel of additional physical 

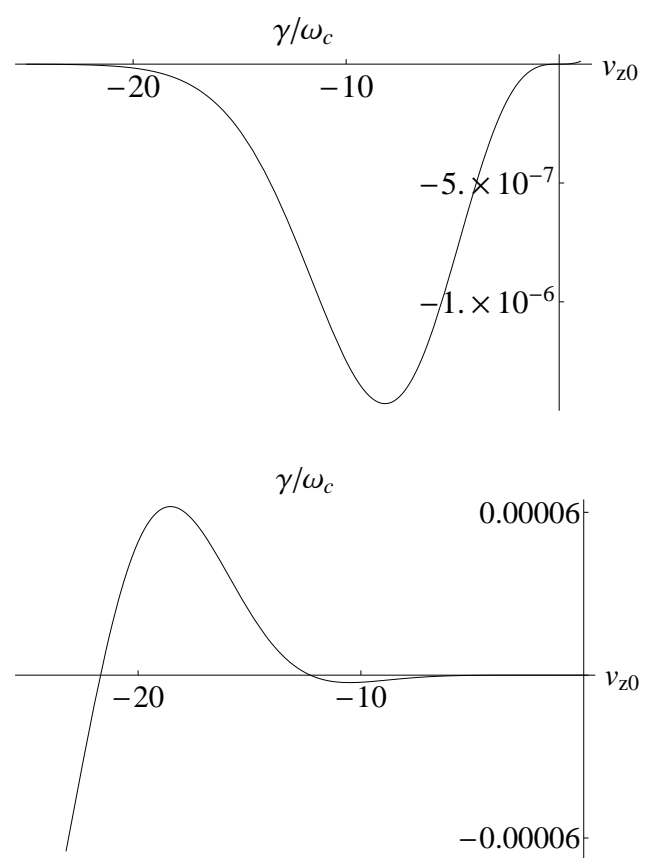

FIG. 11. Variation of $\gamma / \omega_{c}(49)$ with the resonant velocity $v_{z 0}$. (Upper panel) : The parameters are those of Fig. 2. (Lower panel) : The parameters are those of Fig. 6.

processes able to reduce the efficiency of the wave-particles interactions can increase this range of time by one order of magnitude at least.

\section{CONCLUSION}

The paper studies the self-consistent interactions of whistler envelope solitons with electron beams in inhomogeneous plasmas, using a Hamiltonian model of wave-particle interactions where a nonlinear whistler equation involving the beam current is coupled with lower frequency fluid and Newton equations. It allows to describe the parallel propagation of narrowband whistlers interacting with arbitrary particle distributions in magnetized plasmas.

It is shown that the whistler envelope soliton interacts mainly locally with the beam particles, i.e. it does not exchange energy with all the resonant electrons but only with those moving in its close vicinity, contrary to the case of particles' interaction with whistler turbulence. Interactions are efficient not only if the wave-particle resonance conditions on the velocities are fulfilled, but also if the resonant particles satisfy a locality condition. Thus, as the number of resonant particles involved in the interactions with the solitary structure is smaller than in the case of whistler turbulence, the energy exchanged with them by the 
whistler soliton is also smaller. This double condition has a significative impact on the stability of the soliton.

Indeed, estimates based on the numerical simulations show that whistler solitons can

remain stable during $10^{6}-10^{8} \omega_{c}^{-1}$, depending on the physical parameters. Moreover, if the particles moving in the vicinity of the soliton (local particles) are only intermittently in resonance with it or if the free energy of their distribution is reduced due to any other physical effect, it can propagate in the plasma during a range of time increased by one order of magnitude at least, i.e. $10^{7}-10^{9} \omega_{c}^{-1}$, travelling up to a distance of $10^{4}-10^{5}$ times its own width, and even more. On the contrary, the soliton can be totally damped within a time ranging from several thousands of $\omega_{c}^{-1}$ to a few millions, depending on the physical conditions.

During its interactions with the beam, the soliton can either damp and accelerate particles, either absorb beam energy and cause electron deceleration. If the energy exchanges are significative, the soliton envelope is deformed; its upstream front steepens whereas oscillations appear on its downstream side. Weak density inhomogeneities as the random fluctuations of the solar wind plasma have no strong impact on the interactions of the whistler soliton with the resonant particles.

Electromagnetic quasilinear theory has been used in order to provide an estimate of the loss of energy of a soliton interacting with resonant particles. A rather good agreement is found between these analytical calculations and the numerical simulations based on the Hamiltonian model.

\section{ACKNOWLEDGEMENTS}

This work was granted access to the HPC resources of IDRIS under the allocation 2017A0010510106 made by GENCI. This work has been done within the LABEX Plas@par project, and received financial state aid managed by the Agence Nationale de la Recherche, as part of the programme "Investissements d'avenir" under the reference ANR-11-IDEX0004-02. C.K. acknowledges the "Programme National Soleil Terre" (PNST) and the Centre National d'Etudes Spatiales (CNES, France). The work was carried out with the financial support of the Russian Foundation for Basic Research, project No. 16-52-16010-NTSNIL_a. 


\section{REFERENCES}

1

${ }^{2}$ C. F. Kennel, F. L. Scarf, F. V. Coroniti, R. W. Fredricks, D. A. Gurnett, and E. J. Smith, Correlated whistler and electron plasma oscillation bursts detected on ISEE-3, Geophys. Res. Lett. 7(2), 129-132 (1980). doi:10.1029/GL007i002p00129.

${ }^{3}$ F. V. Coroniti, C. F. Kennel, F. L. Scarf, and E. J. Smith, Whistler mode turbulence in the disturbed solar wind, J. Geophys. Res. : Space Phys. 87(A8), 6029-6044 (2012). doi:10.1029/ja087ia08p06029.

${ }^{4}$ O. Moullard, D. Burgess, C. Salem, A. Mangeney, D. E. Larson, and S. D. Bale, Whistler waves, Langmuir waves and single loss cone electron distributions inside a magnetic cloud: Observations, J. Geophys. Res. : Space Phys., 106(A5), 8301-8313 (2001). doi: 10.1029/2000JA900144.

${ }^{5}$ C. Cattell, J. R. Wygant, K. Goetz, K. Kersten, P. J. Kellogg, T. von Rosenvinge, S. D. Bale, I. Roth, M. Temerin, M. K. Hudson, R. A. Mewaldt, M. Wiedenbeck, M. Maksimovic, R. Ergun, M. Acuna, and C. T. Russell, Discovery of very large amplitude whistlermode waves in Earth's radiation belts, Geophys. Res. Lett. 35(1), L01105 (2008). doi: 10.1029/2007GL032009.

${ }^{6}$ O. Agapitov, V. Krasnoselskikh, Y. V. Khotyaintsev, and G. Rolland, A statistical study of the propagation characteristics of whistler waves observed by Cluster, Geophys. Res. Lett. 38(20), L20103 (2011). doi:10.1029/2011GL049597.

${ }^{7}$ L. B. Wilson, C. A. Cattell, P. J. Kellogg, J. R. Wygant, K. Goetz, A. Breneman, and K. Kersten, The properties of large amplitude whistler mode waves in the magnetosphere: Propagation and relationship with geomagnetic activity, Geophys. Res. Lett. 38(17), 17107 (2011). doi:10.1029/2011GL048671.

${ }^{8}$ C. Krafft, P. Thévenet, G. Matthieussent, B. Lundin, G. Belmont, B. Lembège, J. Solomon, J. Lavergnat, and T. Lehner, Whistler wave emission by a modulated electron beam, Phys. Rev. Lett. 72, 649 (1994). doi:10.1103/PhysRevLett.72.649.

${ }^{9}$ M. Starodubtsev, C. Krafft, B. Lundin, and P. Thévenet, Resonant Cherenkov emission of whistlers by a modulated electron beam, Phys. Plasmas 6(7), 2862-2869 (1999). doi: $10.1063 / 1.873244$. 
${ }^{10} \mathrm{M}$. Starodubtsev and C. Krafft, Resonant cyclotron emission of whistler waves by a modulated electron beam, Phys. Rev. Lett. 83, 1335-1338 (1999). doi: 10.1103/PhysRevLett.83.1335.

${ }^{11}$ M. Starodubtsev, C. Krafft, P. Thévenet, and A. Kostrov, Whistler wave emission by a modulated electron beam through transition radiation, Phys. Plasmas 6(5), 1427-1434 (1999). doi:10.1063/1.873393.

${ }^{12}$ R. L. Stenzel, Whistler waves in space and laboratory plasmas, J. Geophys. Res. : Space Phys. 104(A7), 14379-14395 (1999). ISSN 2156-2202. doi:10.1029/1998JA900120.

${ }^{13}$ J. C. Lee and F. W. Crawford, Stability analysis of whistler amplification, J. Geophys. Res. : Space Phys. 75(1), 85-96 (1970). doi:10.1029/JA075i001p00085.

${ }^{14} \mathrm{~K}$. Hashimoto and H. Matsumoto, Temperature anisotropy and beam type whistler instabilities, Phys. Fluids 19(10), 1507-1512 (1976). doi:10.1063/1.861342.

${ }^{15}$ Y. Omura and H. Matsumoto, Computer experiment on whistler and plasma wave emissions for spacelab-2 electron beam, Geophys. Res. Lett. 15,319 (1988).

${ }^{16}$ Y.L. Zhang, H. Matsumoto, and Y. Omura, Linear and nonlinear interactions of an electron beam with oblique whistler and electrostatic waves in the magnitosphere, J. Geophys. Res. : Space Phys. 98(A12), 21353-21363 (1993).

${ }^{17}$ K. I. Nishikawa, O. Buneman, and T. Neubert, New aspects of whistler waves driven by an electron beam studied by a 3D electromagnetic code, Geophys. Res. Lett. 21(11), 1019-1022 (1994). doi:10.1029/94GL00695.

${ }^{18}$ A. Volokitin, C. Krafft, and G. Matthieussent, Whistler waves emission by a modulated electron beam : nonlinear theory, Phys. Plasmas 4(11), 4126-35 (1997). doi: $10.1063 / 1.872532$.

${ }^{19}$ C. Krafft and A. Volokitin, Nonlinear interaction of whistler waves with a modulated thin electron beam, Phys. Plasmas 5, 4243 (1998). doi:10.1063/1.873160.

${ }^{20}$ C. Krafft, A. S. Volokitin, and M. Flé, Nonlinear electron beam interaction with a whistler wave packet, Phys. Plasmas 7(11), 4423-4432 (2000). doi:10.1063/1.1308565.

${ }^{21}$ C. Krafft and A. Volokitin, Interaction of suprathermal solar wind electron fluxes with sheared whistler waves : Fan instability, Ann. Geophys. 21, 1-11 (2003). doi: 10.5194/angeo-21-1393-2003.

${ }^{22}$ V.Y. Trakhtengerts and M.J. Rycroft, Whistler-electron interactions in the magnetosphere: new results and novel approaches, J. Atm. Sol.-Terr. Phys., 62(17-18), $1719-1733$ (2000). 
doi:http://dx.doi.org/10.1016/S1364-6826(00)00122-X.

${ }^{23}$ B. Eliasson and M. Lazar, Nonlinear evolution of the electromagnetic electron-cyclotron instability in bi-kappa distributed plasma, Phys. Plasmas 22(6), 062109 (2015). doi: $10.1063 / 1.4922479$.

${ }^{24}$ M. Hikishima, S. Yagitani, Y. Omura, and I. Nagano, Full particle simulation of whistlermode rising chorus emissions in the magnetosphere, J. Geophys. Res. : Space Phys. 114 (A1), A01203 (2009). doi:10.1029/2008JA013625.

${ }^{25}$ C. Krafft, A. S. Volokitin, and V. V. Krasnoselskikh, Interaction of energetic particles with waves in strongly inhomogeneous solar wind plasmas, Astrophys. J. 778,111 (2013). doi:10.1088/0004-637X/778/2/111.

${ }^{26}$ C. Krafft, A. S. Volokitin, and V. V. Krasnoselskikh, Langmuir wave decay in inhomogeneous solar wind plasmas: Simulation results, Astrophys. J. 809(2), 176 (2015).

${ }^{27}$ H. J. Beinroth and F. M. Neubauer, Properties of whistler mode waves between 0.3 and 1.0 au from Helios observations, J. Geophys. Res. : Space Phys. 86(A9), 7755-7760 (1981). doi:10.1029/JA086iA09p07755.

${ }^{28}$ D. Lengyel-Frey, R. A. Hess, R. J. MacDowall, R. G. Stone, N. Lin, A. Balogh, and R. Forsyth, Ulysses observations of whistler waves at interplanetary shocks and in the solar wind, J. Geophys. Res. : Space Phys. 101(A12), 27555-27564 (1996). doi: 10.1029/96JA00548.

${ }^{29}$ C. T. Russell, D. D. Childers, and P. J. Coleman, Ogo 5 observations of upstream waves in the interplanetary medium: Discrete wave packets, J. Geophys. Res. : Space Phys. 76 (4), 845-861 (1971). doi:10.1029/JA076i004p00845.

${ }^{30}$ G.-L. Huang, D.-Y. Wang, and Q.-W. Song, Whistler waves in freja observations, J. Geophys. Res. : Space Phys. 109(A2), A02307 (2004). doi:10.1029/2003JA010137.

${ }^{31}$ O. Moullard, A. Masson, H. Laakso, M. Parrot, P. Décréau, O. Santolik, and M. Andre, Density modulated whistler mode emissions observed near the plasmapause, Geophys. Res. Lett. 29(20), 361-364 (2002). doi:10.1029/2002GL015101.

${ }^{32}$ A. S. Kingsep, L. I. Rudakov, and R. N. Sudan, Spectra of strong Langmuir turbulence, Phys. Rev. Lett. 31, 1482-1484 (1973). doi:10.1103/PhysRevLett.31.1482.

${ }^{33}$ L. I. Rudakov, Sov. Phys. Dokl [Dokl. Akad. Nauk S.S.S.R. 207, 821 (1972)] 17, 1166 (1973). 
${ }^{34}$ E. I. Valeo and Y.L. Kruer, Solitons and resonant absorption, Phys.Rev. Lett., 33(13), 750-753 (1974).

${ }^{35}$ V.V. Gorev and A.S. Kingsep, Interaction of Langmuir solitons with plasma particles, JETP, 39(6), 1008 (1974).

${ }^{36}$ V.I. Karpman, The effects of the interaction between ion-sound solitons and resonance particles in a plasma, JETP, 77, 1382-1395 (1979).

${ }^{37}$ K. Rypdal, J. P. Lynov, H. L. Pécseli, J. Juul Rasmussen, and K. Thomsen, Interaction of Langmuir solitons with resonant particles, Phys. Scripta, 1982(T2B), 534 (1982).

${ }^{38}$ M. Y. Yu, P. Shukla, and K. Spatschek, Interaction of an electron beam with whistler solitons, Phys. Rev. A, 14, 1547-1550 (1976). doi:10.1103/PhysRevA.14.1547.

${ }^{39}$ Y. Omura and H. Matsumoto, Competing processes of whistler and electrostatic instabilities in the magnetosphere, J. Geophys. Res. : Space Phys., 92(A8), 8649-8659 (1987). doi:10.1029/JA092iA08p08649.

${ }^{40}$ T. F. Bell and O. Buneman, Plasma instability in the whistler mode caused by a gyrating electron stream, Phys. Rev., 133(5A), A1300-A1302, (1964).

${ }^{41}$ R.A. Dory, G.E. Guest, E.G. Harris, Unstable electrostatic plasma waves propagating perpendicular to a magnetic field, Phys. Rev. Lett. 7(14), 131 (1965). doi: 10.1103/PhysRevLett.14.131.

${ }^{42}$ C. Krafft and A.Volokitin, Dynamics of whistler enevelope solitons in inhomogeneous plasmas, Phys. Plasmas, in press, 2018.

${ }^{43}$ C. Krafft and A. Volokitin, Nonlinear fan instability of electromagnetic waves, Phys. Plasmas, 17, 102303 (2010). doi:10.1063/1.3479829.

${ }^{44}$ R.Z. Sagdeev and A.A. Galeev, Nonlinear Plasma Theory, W.A. Benjamin, Inc., New York, 1969. 